\title{
High-resolution Temporal Representations of Alcohol and Tobacco Behaviors from Social Media Data
}

\author{
TOM HUANG, University of Waterloo \\ ANAS ELGHAFARI, New York University \\ KUNAL RELIA, New York University \\ RUMI CHUNARA, New York University
}

\begin{abstract}
Understanding tobacco- and alcohol-related behavioral patterns is critical for uncovering risk factors and potentially designing targeted social computing intervention systems. Given that we make choices multiple times per day, hourly and daily patterns are critical for better understanding behaviors. Here, we combine natural language processing, machine learning and time series analyses to assess Twitter activity specifically related to alcohol and tobacco consumption and their sub-daily, daily and weekly cycles. Twitter self-reports of alcohol and tobacco use are compared to other data streams available at similar temporal resolution. We assess if discussion of drinking by inferred underage versus legal age people or discussion of use of different types of tobacco products can be differentiated using these temporal patterns. We find that time and frequency domain representations of behaviors on social media can provide meaningful and unique insights, and we discuss the types of behaviors for which the approach may be most useful.
\end{abstract}

CCS Concepts: • Information systems $\rightarrow$ Web and social media search; Content analysis and feature selection; • Mathematics of computing $\rightarrow$ Time series analysis;

Additional Key Words and Phrases: Natural language processing; time-series; behavior; social media; health; alcohol; tobacco

ACM Reference Format:

Tom Huang, Anas Elghafari, Kunal Relia, and Rumi Chunara. 2017. High-resolution Temporal Representations of Alcohol and Tobacco Behaviors from Social Media Data. Proc. ACM Hum.-Comput. Interact. 1, CSCW,

Article 54 (November 2017), 26 pages. https://doi.org/10.1145/3134689

\section{INTRODUCTION}

Time and seasonality are important concepts in health. Temporal patterns of health outcomes, behaviors and risk factors have always been used to help inform focused prevention and intervention efforts at individual, community and policy levels. In particular, as we move to a more precision-based era of public health, high-resolution (hourly, daily) measures can help improve public health efforts at multiple levels, beyond what is currently possible. For example, high-resolution information about when targeted behavior patterns are likely to manifest can be used in the design

This work is supported in part by the National Institutes of Health, under grants R21AA023901 and R21MH110190 and a grant from the Truth Initiative.

Authors' addresses: T. Huang, Department of Statistics and Actuarial Science, University of Waterloo; A. Elghafari, K. Relia, Tandon School of Engineering, New York University; R. Chunara, Tandon School of Engineering and College of Global Public Health, New York University;

Permission to make digital or hard copies of part or all of this work for personal or classroom use is granted without fee provided that copies are not made or distributed for profit or commercial advantage and that copies bear this notice and the full citation on the first page. Copyrights for third-party components of this work must be honored. For all other uses, contact the owner/author(s).

(C) 2017 Copyright held by the owner/author(s).

2573-0142/2017/11-ART54

https://doi.org/10.1145/3134689

PACM on Human-Computer Interaction, Vol. 1, No. CSCW, Article 54. Publication date: November 2017. 
of novel social computing systems to inform the right times at which to deliver an intervention, or target groups or places at high risk for unhealthy behaviors (e.g. a text message to encourage maintenance of smoking cessation or impulse control at trigger times and places) [38]. Also, public health policies can be shaped based on this data, such as the recent efforts to restrict hours and days of alcohol sales to reduce excessive consumption and related harms [14, 49, 58]. In general, for a view of health and medicine that encompasses both biological and behavioral components, it is important to gain insight into behavioral patterns and how we can best design systems to help recognize and target those that are risky.

Issues such as binge drinking, addiction and new and alternative tobacco products make alcohol and tobacco use behaviors prominent public health concerns today. The increasing magnitude and cost of alcohol and tobacco related problems make them especially vital behaviors to monitor, identify high-risk groups and patterns of consumption during typical weeks as well as changes during holiday or other periods and moments for most effective implementation of interventions [42]. Total costs to federal, state and local governments of substance use disorders, which most often have their onset in adolescence, are at least $\$ 468$ billion per year which is almost $\$ 1,500$ for every person in America [61]. Further, many alcohol and tobacco behaviors are both public and social. As such, they will often be shared and discussed on social media (illustrated by numerous other studies examining topics and locations of social media discussion about tobacco and alcohol $[7,20,50])$. Simultaneously, tobacco use and addiction mostly begin during youth and young adulthood [78] and alcohol use is associated with the top four leading causes of morbidity and mortality in adolescents [33] making them significant health risks for this age group. National health surveillance programs such as the National Survey on Drug Use and Health (NSDUH) and the National Youth Tobacco Surveys (NYTS) are denominator-based surveillance systems that provide population-representative structured data to inform policy and guide clinical and research objectives. However, survey time frames and sampling frames do not support real-time monitoring, robust subgroup investigation, flexible follow-up or feedback of health alerts and information to subjects. Further, there is an awareness in the public health community that existing estimates from survey-based reports of use do not reflect the full extent of variability of drinking over limited time periods, suffer from under-reporting especially in regard to substance use, and do not adequately represent consumption during all time periods in the calendar year [42].

The Internet, and specifically social media, is an increasingly profuse source of high temporal resolution information. Based on work in the social computing community, social media data have been used to study many public health topics including diet [1], fitness [83] and mental health [27]. Beyond discussion about topics, the images and text in social media data can also provide firsthand information about individuals engaging in particular behaviors (e.g. actual time and location-linked instances of consuming alcohol or tobacco, versus online searches or check-ins to a bar or hookah bar which don't specifically indicate consumption [39]). However, it must be made clear that without any offline, linked validation study, only communication about behaviors can be studied. Even so, these discussions about behaviors are still very important when considering health as a comprehensive framework that includes communication of health-related topics, risks and behaviors. Just as there are benefits drawbacks to survey-based reports, there are challenges and also specific aspects of social media communications of behavior that can complement and augment existing surveillance approaches. Assessing high-resolution patterns of behavior on social media can provide empirical understanding of when people share information about behaviors, and can be used in socio-ecological frameworks of behavior which consider the social norms and other factors which may moderate substance use.

Knowledge about when and where people make decisions brings with it a lot of actionable information, and is a common theme in computer supported cooperative work. This need for 
understanding time-dependent parameters is evident in the design of many computer supported systems such as workflow technologies for health interventions [12] and for temporal coordination $[13,70]$. Temporal patterns have also been recognized in the design of chronic care systems by accounting for cycles [19] and incorporating on-body sensors and machine learning to infer people's activities and encourage positive behavior [24]. These advances highlight the potential to deliver health behavior interventions tailored not only in a personalized manner but also to a person's frequently changing behaviors and environments. The need for contextual, dynamic and stratified understanding of behavior is critical towards the development of "just-in-time" interventions; an active area of research for behaviors like smoking and drinking to support cessation or impulse control $[8,52]$. Towards this end, we consider the temporal representations of behavior from social media communications focusing on alcohol and tobacco use cases.

Beyond point values about a targeted behavior communication, there are many reasons to explore other representations. First, any effort to evaluate how similar or different time series are will depend on the selection of meaningful features. Second, incidence of Tweets about a particular behavior (point value at a specific time) is affected by three factors: natural language processing (classification) error, sample confidence intervals, and communication trends regarding the particular behavior on a social media platform. Thus, it could be more informative to measure temporal patterns of communications (if we assume that classification error is constant across different hours and days, then the relative differences and temporal cycles highlighted by the time-series analyses should persist withstanding classification error). Recognizing that temporal representations are useful the question arises: which time periods of behavior communications are informative? And further, how do those temporal patterns resolve for different groups and about different sub-topics?

In concert with consideration for population generalizability and denominator challenges [22], the nature of social media presents flexibility, nuance and timeliness alongside high use by youth, amplify the potential of social media as a complementary surveillance approach especially suitable to tobacco and alcohol behavior studies for these types of questions. Therefore, we use alcohol and tobacco consumption as use cases by which to explore what kinds of high frequency representations (diurnal, day-to-day or weekly patterns) resolved from social media data can be used to evaluate meaningful differences in these temporal patterns of behavior communication. While the analyses are demonstrated using alcohol and tobacco behavior communications, combining data mining and statistical analysis we develop a set of computational steps that can enable new insight into other communication about other behaviors as well, broadly regarding 1) appropriate temporal representations of behavior communications on social media, 2) differences in communication by different groups and 3) communication about different types of behavior. The specific research questions we examine here are:

- Which temporal (time and frequency domain) representations of social media behavior communications are suitable for illustrating and differentiating hourly, daily or weekly patterns?

- Does the self-reported alcohol consumption by underage users on social media exhibit a different temporal pattern than self-reported consumption by users of legal age?

- Do self-reported consumption activities follow different temporal patterns for different tobacco products (i.e. hookah versus other tobacco products)?

\section{RELATED WORK}

Relevant related research include efforts on (1) mining social media for health behavior-related insights and associations, (2) examining seasonal variations in clinical and laboratory variables (3) studying temporal trends and periodicity in human generated data from weblogs, call data records 
and social media, (4) studying patterns in behavior especially regarding substance use, and (5) temporal data mining approaches. We review each of these areas here.

Some aspects of health-related behaviors are beginning to get examined by mining social media data. Especially in the social computing community, large data mined from online sources has been used to study many aspects about human behavior. Specifically, this community has realized that social media data can be useful for identifying personal state and experience [51, 59]. In regards to health related behavioral insight, topics considered are typically those conducive to being discussed on social media and which may not be disclosed or reported elsewhere. For example, for predicting mental illness severity in pro-eating disorder communities [17], or evaluating aspects of subgroups of those with mental health, sleep or drug issues [15, 29, 57].

Overall, seasonality in health has been considered in topics such as birth weights, viral infections, mental health, anxiety and depression, mortality and epidemic waves [11, 25, 45, 63, 82]. In general the temporal effects considered have been at monthly or yearly time-scales, either based on the nature of the patterns (infectious disease cycles which happen over the course of seasons and years) or the mode of data collection. Survey-based data gathering measures have inherent time and labor costs and are affected by recall and information bias. All these factors limit how frequently data can be collected [73]. Simultaneously, the behavioral science community has been stressing the need for contextual, dynamic ways to quantify understanding of behavior [71, 81]. Measures derived using mail, telephone or paper questionnaires are robust, yet can introduce forms of self-report bias and can suffer from issues of recall and time commitment [85].

Using Internet-sourced data, work has been done on characterizing topics expressed in weblogs or search queries, including behaviors (again at the seasonal/monthly level). The seasonal component of mental health patterns has been assessed via wavelet analysis on Google search queries [6] Additionally, prediction of many behaviorally-relevant outcomes such as unemployment from search logs, shifts in suicidal ideation and time-dependent concerns about pregnancy and childbirth have been generated from search logs [27, 37, 69]. Higher-resolution trends (daily) have been ascertained from social media as a common theme, however have been focused on infectious disease surveillance [16, 21, 77]. Some work using social media and has examined mental health spatially [26] or over time [41]. Other work has used verbal (text) expressions of specific real-world activities from Twitter, extracted based on finding words that correlate to temporal patterns from Foursquare check-ins. Perhaps closest to the work here, this specific study was focused on diurnal patterns only, and specific natural extensions recommended from the work included developing deeper natural language processing methods to better isolate individuals engaged in behaviors as well as examine the non-periodic components of the signals as well as frequency analysis to allow for drift over time [46].

Specifically in relation to alcohol-related behaviors, patterns of consumption are important to resolve to better understand behavior at specific time-scales and discern temporal relations with other phenomena such as traffic fatalities and homicide, in which alcohol use is known to play a significant role [4]. Some historical studies using survey methodology have uncovered general patterns at the level of days, linking consumption levels by day in the week to frequency of alcohol-related traffic fatalities [4,36]. Alcohol consumption patterns over the course of a single day are also important to consider for a host of bio-psychosocial factors. It has been shown that evening alcohol consumption can affect glucose control in diabetics [86]; daytime consumption can interfere with work performance [55]; and driving and accidents are associated with morning consumption [67]. For tobacco, the types of products used is also a growing area of interest as new products emerge on the market and their impact on health, associated behavior patterns, and short-term and long-term risks are unexplored. Initial investigative studies have shown that the public understands various tobacco products to confer significantly different levels of risk and 
benefits, and also have different normative behaviors. This has indicated that studies of the risks of newer tobacco products (specifically hookah and e-cigarettes) are needed [72]. Understanding patterns of alcohol and tobacco use at the hourly and daily level can also be helpful to understand and better focus substance use prevention and interventions. Drawing inference about temporal patterning has been continually hindered by insufficient and homogeneous samples from surveys

Finally, temporal data mining is the general area of data mining which focuses on data that contains temporal information [3]. The ultimate goal of temporal data mining being discovery of any latent relations or those simply not represented by any other data sources. Generally, there are three components to the process: first the representation and modeling of the data sequence in a suitable format, then definition of similarity measures between sequences and finally third, the application of models to the actual mining problem. While high resolution methods for behavioral-health data are nascent, autoregressive methods have commonly been used in other applications such as derivative and stock prices (daily). Such methods have also been applied more readily in healthcare through complex data acquisition techniques like electrocardiograms and other physiological measures [48]. Gaussian processes and Markov models have started to be used more recently for statistical modeling and prediction of continuous data such as from time series [40], however the use of these approaches hinges on selection of representations of the data most appropriate for the underlying process. Thus, before any work on temporal prediction can be accomplished, the temporal "features" of the data that represent relevant differences must be identified.

\section{DATA PREPROCESSING}

\subsection{Sampling}

Twitter data from two different time periods (June and September 2015) was selected to examine behavioral representations at different times of year. We used geo-located data (polygon) from the Twitter API, and restricted to data only from USA, resulting in approximately 50 million Tweets per month. Stop words, Retweets and URLs were removed, and all characters converted to lower case per convention in Twitter data processing [26, 88]. A few intervals of hours in the 3rd week of September had missing data (considered missing if there were fewer than 5000 Tweets in an hour). Missing data was filled by Last Observation Carried Forward (LOCF).

\subsection{Classification}

For both alcohol and tobacco (the "behaviors"), two separate supervised-learning n-gram based text classification pipelines were developed. For each of the behaviors, Tweets were classified hierarchically into behavior versus not behavior related, then first person versus not first person (the person Tweeting is talking about themselves performing the action). Finally, to isolate episodes of consuming alcohol or tobacco that are linked to precise times, for each pipeline the Tweets were classified into past, present, and future following previous work identifying behavior stages from social media [53]. As we are interested in self-reported communications of behaviors linked to time, we used data from the "present" category of the last classification for further analyses in this paper. A challenge in parsing tobacco-related Twitter postings is the common occurrence of habitual posts (e.g. "As bad as it is, a cigarette before bed always calms me"). For the purposes of this work, we combined present and habitual tobacco posts.

We implemented the classifiers using the scikit-learn package in Python [66] and a randomized grid search was used to optimize all hyperparameters of the classifiers at each stage of the hierarchy. For classification at each stage we considered a logistic regression (LR), support vector machines (SVM) and random forest (RF) approach. Features included were n-grams of length 1, 2 and 3. We used word n-grams as has been done in other social media classification work [50]. We found that 
using character n-grams did not improve performance, perhaps due to the medium-sized training data set.

Parameter space for the regularization parameter was a uniform distribution across $\left(10^{-4}, 10^{4}\right)$. For each of the classifiers (behavior, first person, and past/present/future), after completing a hyperparameter grid search on each approach we decided to use logistic regression (LR) with L2 regularization. Regularization coefficient was $C=0.01$. Logistic regression is a well-established classification algorithm which is appropriate if the data can be assumed to be linearly separable, efficient to run and output is easily interpretable as a probability.

\subsection{Labelling Procedure}

Creating the training data set was a challenging part of identifying present Tweets due to Internet slang and, for the tobacco classification pipeline, due to the overlap between tobacco and marijuana terms. Tobacco use is often difficult to disentangle semantically from marijuana (e.g. "Maybeeeeee I should start smoking again and forget everything else because life sucks atm"). Thus labeling was performed using a combination of efforts by Amazon Mechanical Turk (AMT) workers and our team members.

For both behaviors, due to the low percentage of behavior-specific Tweets, as well as the nuanced differences between marijuana and tobacco, the training (labelled) data was very carefully curated and we used active learning following the approach in [53]. First, we developed a dictionary, using Internet sources such as Urban dictionary and Wikipedia as references to pre-filter Tweets that would be submitted for manual labeling, in order to increase instances of target classes. Through careful manual and iterative examination of Tweets, as well as using Internet references, we devised and tested criteria for the labeling. This included criteria to exclude terms that were not related to alcohol or tobacco (e.g. including those that represented marijuana) as well as detailed instructions along with specific examples of what we meant by a present Tweet versus future, or tobacco versus marijuana. The resulting criteria were then outlined in the instructions on AMT and used by our team as reference. We ran several test iterations on AMT, submitting Tweets and evaluating the resulting labels by hand with our team, and iterating on the instructions. The labeling itself was also done in an iterative manner. After each group of Tweets was labelled, we would examine the agreement cases as well as the disagreement cases, and use this to tweak the instructions and consider the filtering keywords. We cross-referenced Internet sources to adjudicate labeling disagreement instances for Tweets with obscure terms and slang. This check also resulted in removal of labels by AMT workers who were not self-consistent in their labels. In particular, we also re-labelled data at the decision boundary by hand in order to improve performance of the classifier. This approach also helped us find Tweets to label given the low prevalence of each behavior. In sum, every label used for training of the classifiers was either agreed-upon by two labelers, or manually reviewed and adjudicated by one of the authors.

The balance of training and test data also had to be optimized to include as many example Tweets but not overfit the model (with too many "positives"). For tobacco classification this resulted in 26,160 labelled Tweets (14\% were labelled as tobacco), 3,670 labelled Tweets for first-person classification (44\% positive first person) and 1,620 labelled Tweets for present (59\% positive). Using a 10 -fold cross-validation, F1 scores for the behavior, first-person and present classifiers were: 0.87 , 0.75 and 0.81 (alcohol) and 0.86, 0.98, 0.75 (tobacco). Accuracy was $0.83,0.77$ and 0.71 (alcohol) and 0.96, 0.77 and 0.77 (tobacco). We also note the area-under-curve (AUC) value for these classifiers, corresponding to the receiver operating characteristic (ROC) curves for each classifier to be high: $0.88,0.73$ and 0.78 (alcohol) and $0.99,0.85$ and 0.86 (tobacco). Performance (precision and recall) on non-behavior classes was higher (0.98) due to the higher proportion of such instances, though performance on our classes was still reasonable. Issues of noise/misclassification are highlighted 


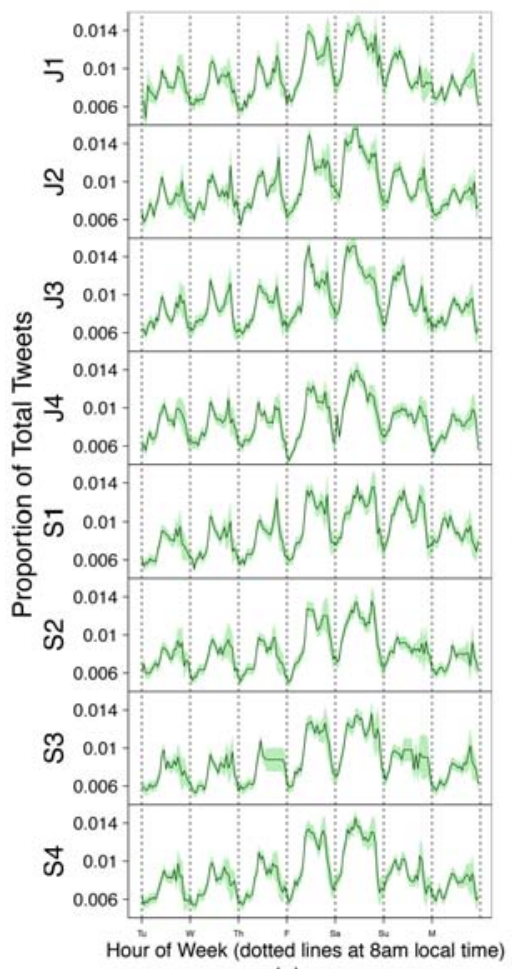

(a)

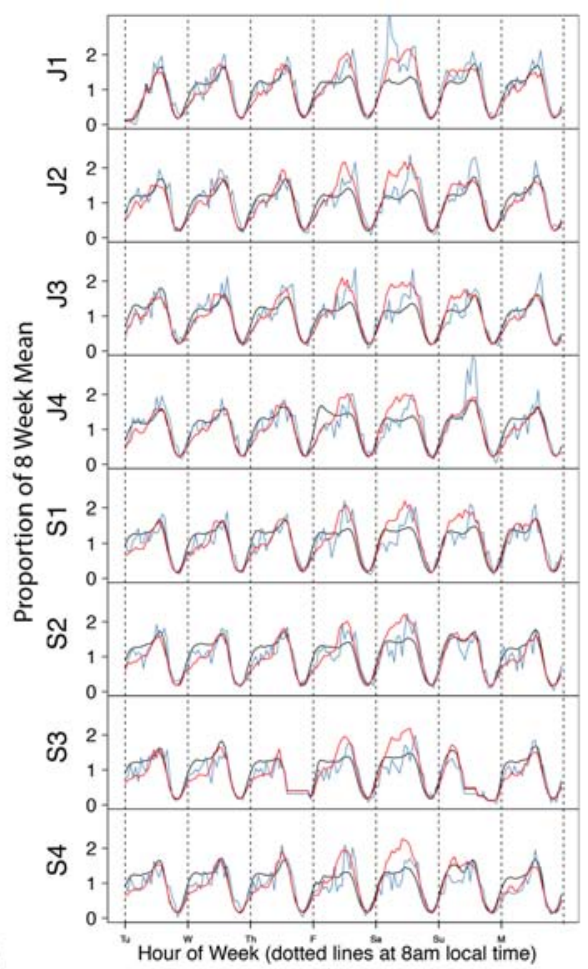

(b)

Fig. 1. (a) Time series by week of first-person alcohol consumption discussion for June (J1 to J4) and September (S1 to S4). The line represents the mean value (proportion of present alcohol Tweets out of all Tweets at each hour), shaded area around time series are 95\% confidence intervals. (b) Time series of all Tweets (black), alcohol consumption (red) and tobacco consumption (blue) for weeks in June (J1 to J4) and September (S1 to S4). Each time series is normalized by its mean over all 8 weeks. Dotted lines are at 8 am local time. Missing data from S3 visible as flat lines.

further in the Discussion section - if assumed to be constant over time, these issues should not affect the temporal trends. Top features for each classification stage and their associated feature strength $\beta$ are described in Table 2 in the Appendix. As we used the approach from [53] for the alcohol classification, labelled data distributions and classifier performance were the same as results from that study.

3.3.1 Age Classification. There are multiple methods in the literature that have been used to infer the age of Twitter users with reasonable accuracy. Sloan et al. [79] discussed how vocabulary used in tweets can help in determining a user's age. Our model harnessed this approach to classify the accounts of the included Tweets into underage $(<21$ years) or legal age $(\geq 21)$ categories. We performed age classification on the alcohol-related data set to analyze how inferred underage and legal age people communicate self-reports about drinking patterns.

In this approach we collected a training set of data constructed from users who wished their own birthday in the previous month. This was done by searching for Tweets having the keywords "HappyBirthdayToMe N years" or "mybirthday N years" or "It's My Birthday" + "N years" or "happy $\mathrm{Nth} / \mathrm{st} / \mathrm{nd} / \mathrm{rd}$ birthday to me" where $\mathrm{N}$ ranged from 10 to 70 [2]. Out of a total of 1025 Tweets, 956 
Tweets were left after removing duplicates. For each of these 956 now unique users, their past 100 Tweets were fetched. From this pool of Tweets, all re-Tweets (those Tweets that contain "RT") and Tweets containing a URL were removed. Further, we ensured that all included Tweets were in English by checking that they contained at least two stop words. Finally for the age training set we were left with 61,596 Tweets, out of which 29,138 were from legal age users and 32,458 were from underage users. For the age classification, we used uni-gram features from the text, and Support Vector Machine classification with a linear kernel (regularization parameter 10), which have shown to perform well for such binary classifications [2]. F1 score of the classifier was 0.82 . Though this approach of using inference to classifying Twitter users by demographic has precedence in the literature [26], we note that the approach can be biased towards users who self-report their age. If the age classification error is consistent over time, it may affect point values, but the temporal trends and their differences would be consistent. In any case, the age trends may not be representative of the general population and we discuss the need for a future study to systematically assess and connect results to offline groups in the Discussion section.

3.3.2 Tobacco Product Identification. We focused on hookah as it is an increasingly popular alternative tobacco product with important health-related consequences [56]. Another distinguishing characteristic for hookah (versus traditional cigarettes or vapes) is that it is often consumed in a social setting (with peers or friends). Thus, we would hypothesize that the use of hookah may be communicated on social media through different patterns than other products. Based on inspection of a large number of tobacco-related Tweets by our team, we developed a list of keywords to identify use of hookah (Appendix). The keyword filtering was applied only after classification of first-person present Tweets, thus ensuring we are identifying Tweets about present (or habitual) use of hookah.

\section{DATA REPRESENTATIONS}

Any effort to assess how similar or different temporal representations of behavior are, will depend on the selection of meaningful features. Thus we isolate and examine features that represent magnitude and phase in the time domain, as well as the frequency domain. We use frequency and time-based representations (through amplitude, phase and wavelet analyses) of processed social media data. Accordingly we employ methods from time series data mining and clustering to assess how those representations are similar or dissimilar for data generated by different groups (under and legal age people) and consumption of a specific product type.

\subsection{Time Series}

First, we generated and examined the temporal pattern of Tweets about alcohol and tobacco behavior (from the output of the "present" classifier), linked to hourly times throughout each week of both months in our data. In order to account for trends of Twitter posts in general, we normalized by the total number of Tweets at an hourly resolution. In order to compare data between months, and to ensure no discrepancies based on time zones, we used exactly 28 days from each month, starting on the first Tuesday of each month (September 12015 was on a Tuesday). This resulted in one observation per hour for 4 weeks in each of June and September.

Let $\mathbf{x}=x(t), t=0, \ldots, n-1$ represent the equally spaced observations, with total length equal to the number of hours in 28 days (672). The time-series are subdivided into subsets of length $w=24 \times 7$ (weekly) or $d=24 \mathrm{x} 1$ (daily) for comparison. We consider each day to span the period from $8 \mathrm{am}$ one morning to 8am the next day to capture and group the drinking patterns for a particular day. Overall both the trends of alcohol and tobacco self-reported present consumption Tweets are different from the general Twitter temporal trends (Figure 1b). From these time series we can qualitatively assess 
some temporal features of the data. First, there is a clear daily periodicity to Tweets related to alcohol drinking behavior. The alcohol time series has more prominent diurnal trends than overall Tweets (illustrated in Figure 1a). The proportion of alcohol-consumption Tweets generally increases in the late afternoon peaking at $8 \mathrm{pm}$, and then decreases before midnight. There is a second peak about 8 hours after the first peak in the evening (not as prominent on weekends) most likely due to the fact that overall Tweeting is low at that time of morning increasing the relative proportion of alcohol consumption Tweets. Tobacco consumption Tweets follow a similar diurnal pattern, however not as pronounced. This could be due to the lower proportion of tobacco-consumption Tweets, or a more temporally consistent behavior (people tend to smoke and communicate about smoking more consistently throughout the week while people drink alcohol and communicate more so about drinking at certain times of day or week). Further of note is that overall we found a much higher number of alcohol Tweets compared to tobacco Tweets (discussed in the Results section).

We also can qualitatively describe day-to-day trends. In Figure 1a we see that the proportion of alcohol consumption Tweets increases earlier on weekends. The highest proportion overall is reached on Friday and Saturday. Also, accounting for the temporal trends of all tobacco consumption posts, hookah consumption Tweets increase on Friday and Saturday relative to all tobacco consumption reports (Figure 2). Day-to-day trends can also be visualized for specific subgroups. For time series representation of the age-classified alcohol data, we examine the underage and legal age weekly trends of presently drinking tweets from June 2015 (Figure 3). Each series was normalized by the L2-norm of the total number of Tweets that week. Because the total number of Tweets from legal or underage users was not possible to reasonably compute (would require classification of all the Tweets), we normalized over Tweets from each week by the age group in question to make underage and legal age trends comparable. For the age-classified term series, there are some clear differences in the proportion of first-person alcohol consumption Tweets over the course of each day. Tweets from inferred underage people generally are in higher proportion than those from inferred legal age people early in the day on weekdays, while on weekends inferred legal age Tweets are higher over the course of the day consistently for all weeks in June. Due to the high number of data points and noise, it is hard to visually compare all of the days in just the time series, indicating the need for a more summative metric. For this purpose we assess similarity and clustering measures in subsequent sections.

4.1.1 Time Series Comparisons. Though other measures of substance use behaviors at the same high-resolution (hourly, daily) do not exist, we can triangulate with other data sources that are at the same resolution to gain some understanding of how accurately the social media communications may connect to offline behaviors. For this, we used data from Google Trends and Venmo.

Google Trends has been used previously to understand daily and sub-daily patterns for topics such as infectious disease incidence and trading behavior in financial markets [68, 75]. From Google Trends, we selected the topics of: "beer", "wine" and "liquor" (for alcohol comparison) and "hookah" (for hookah comparison), and "cigarette" and "electronic cigarette" (for tobacco, non-hookah comparison). Per Google, topics are a group of terms that share the same concept, in any language [43], thus we used these search terms to include types of alcohol comprehensively just as with the Twitter data. From Google Trends we were able to download data for the same two time periods of the Twitter data (June and September 2015) from the API [44]. All trends were limited to data from the United States to match the Twitter data.

Though the trends from Google can be at a comparable hourly and daily resolution, they only represent online searches and it is not clear a priori if these are indicative of consumption at the linked time (it is possible someone may search for something, and then later act on it or 
consume something related). So, as an additional data source we used transactions from the peerto-peer transaction platform Venmo, which is very popular in the United States [23]. We used four weeks of Venmo data from June 8th to July 5th 2017 from the Venmo API [87], only including person-to-person transactions (discarding transactions in which either of the participants had the "is_business" boolean attribute true). Venmo is only available in the United States. To identify transactions specifically related to alcohol or tobacco, we generated a set of keywords that would be as comprehensive as possible, based on our own experience with Twitter data as well as by using urban dictionary and Wikipedia as references. We list all keywords and emojis used in the Appendix. For Venmo, emojis were essential as we found that from the data gathered, $53.9 \%$ of all Venmo transactions only had emojis in their description and $14.8 \%$ used both text and emojis. The keywords were used to select transactions based on the description field (each Venmo transaction has a description field in which the user who pays or requests the transaction can describe what it is for).

\subsection{Discrete Fourier Transform}

Next, in order to assess the periodic component of the time series quantitatively, we examined the frequency domain representation of each above time series (weekly and monthly). This analysis can therefore be used to answer questions such as, what are the major periodic components of communications about a behavior on social media? And, is the periodic component useful in distinguishing between the behavior communications of different subgroups or on different topics?

We used a Discrete Fourier Transform to quantitatively assess the periodic components. The Fast Fourier Transform (FFT) is used to calculate Discrete Fourier Transform (DFT) coefficients for frequency components in a time series. We also chose the DFT because it is the most well-known method for assessing frequency components, its code is readily available, it is fast to compute, and it does a good job of concentrating the time series energy in the first few coefficients (and we would expect the first few coefficients to be dominant for the time series in question). We also

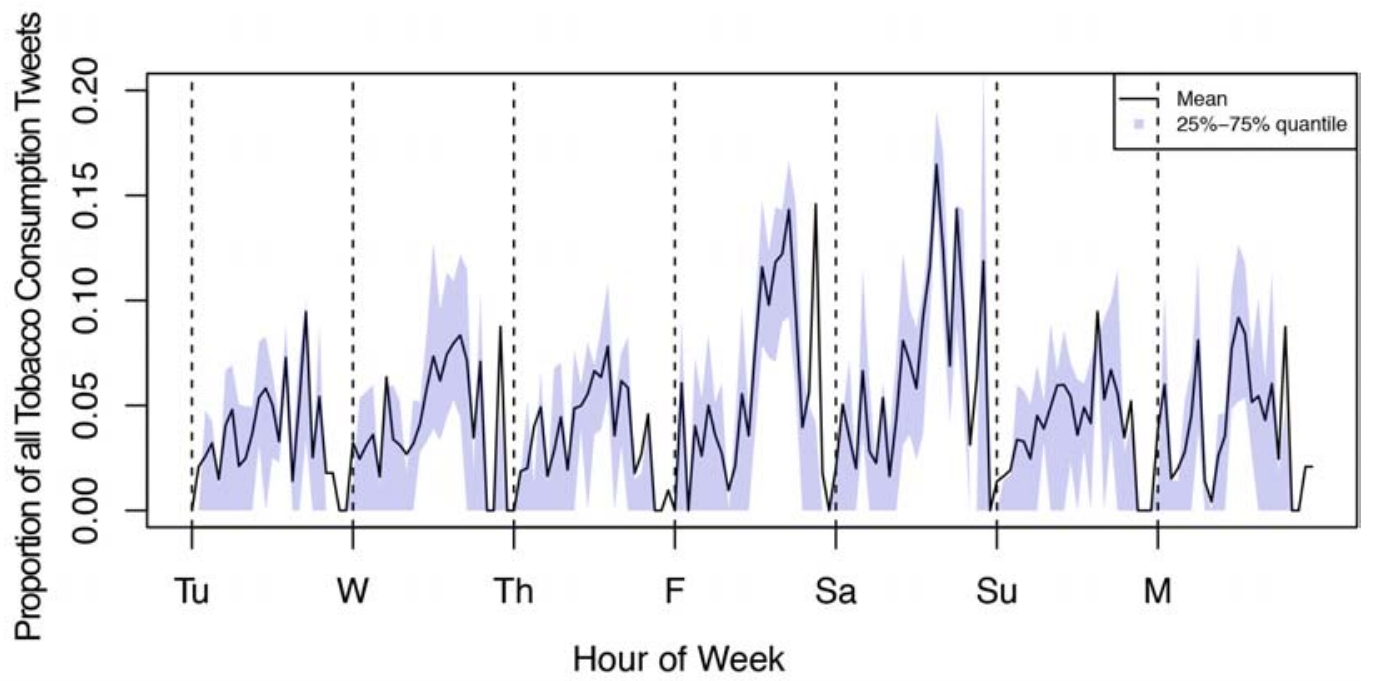

Fig. 2. Time series of hookah consumption Tweets as a proportion of all Tobacco Present Tweets average by day for June and September 2015. Dotted lines at 8am. As the line represents mean over all weeks, quantiles are used instead of confidence intervals. 


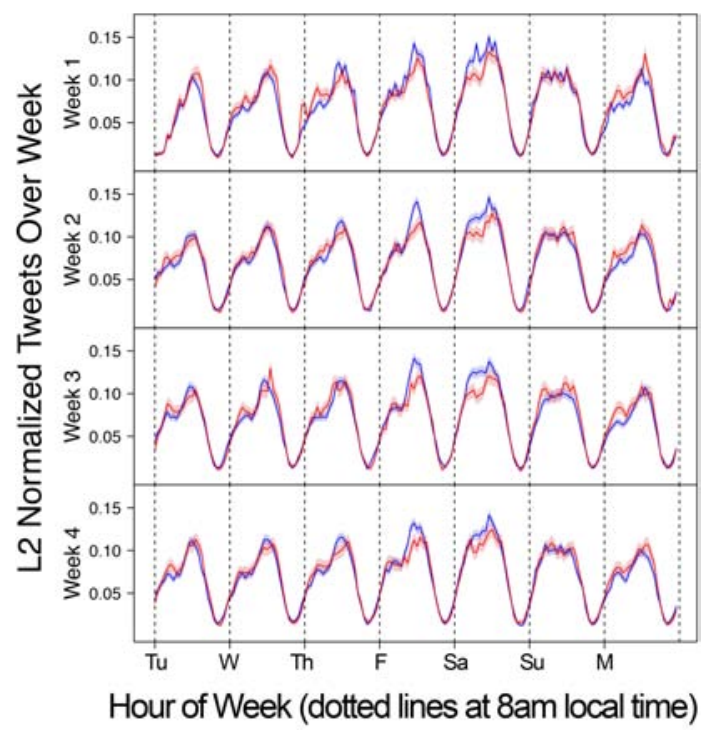

Fig. 3. Time series of drinking posts by inferred legal (blue) and underage (red) people for each week in June 2015. Dotted lines at $8 \mathrm{am}$. Shaded areas represent $95 \%$ confidence intervals.

acknowledge the DFT assumption that frequency components of the series are stationary. From the time series (Figure 1a), we know that the components corresponding to daily and weekly periods are stationary throughout time, thus the Fourier transform is appropriate. While details of the Fourier transform can be found elsewhere [62], we outline the method briefly here.

The $n$-point DFT of a vector $\mathbf{x}=x(t), t=0, \ldots, n-1$ is a vector $\mathbf{X}=\mathbf{X}(\mathrm{f})$ of complex numbers where $f=0,1, \ldots, n-1$ given by:

$$
X(f)=\frac{1}{\sqrt{n}} \sum_{t=0}^{n-1} x_{t} \exp (-j 2 \pi f t / n)
$$

( $j$ is the imaginary unit $j=\sqrt{-1}$. The inverse transformation gives:

$x(t)=\frac{1}{\sqrt{n}} \sum_{f=0}^{n-1} X_{f} \exp (j 2 \pi f t / n)=\frac{A_{0}}{2}+\sum_{k=1} A_{k} \cos \left(k \omega_{1} t-\theta_{k}\right)$ and thus the amplitude of the frequency component $k$ is $M_{k}=\sqrt{A_{k}^{2}+B_{k}^{2}}$ and phase is $\theta_{k}=\arctan \left(\frac{B_{k}}{C_{k}}\right)$.

The DFT can be used to compare the amplitude and phase shift of frequency components of different time series. We first take the DFT of the entire time series and project it onto the frequency basis in question. For example, when comparing the daily frequency component across different weeks, we take the DFT of the entire week's observation vector and examine the $7^{\text {th }}$ component corresponding to a daily frequency (repeats 7 times per week). The complex argument of that coefficient is a representation of the phase of the daily frequency of that week. Thus the DFT can be used to compare the phase of different frequencies (time series). We resolve and compare the amplitude and phase components for the 1st frequency component (weekly) of different days of the time series, to assess how the periodicity (daily component of the alcohol consumption Twitter time series) changes on a day-to-day basis. 


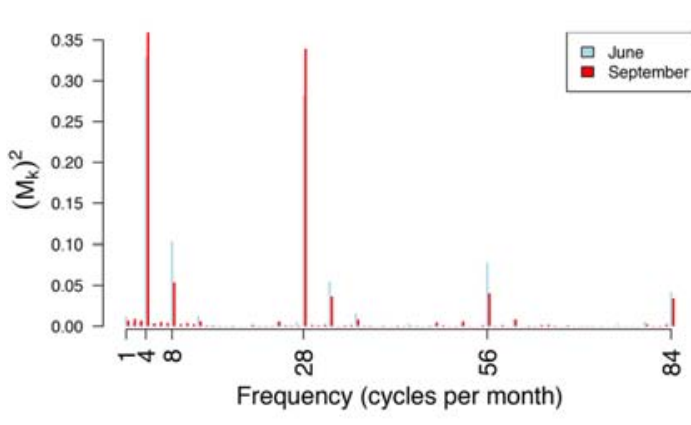

(a)

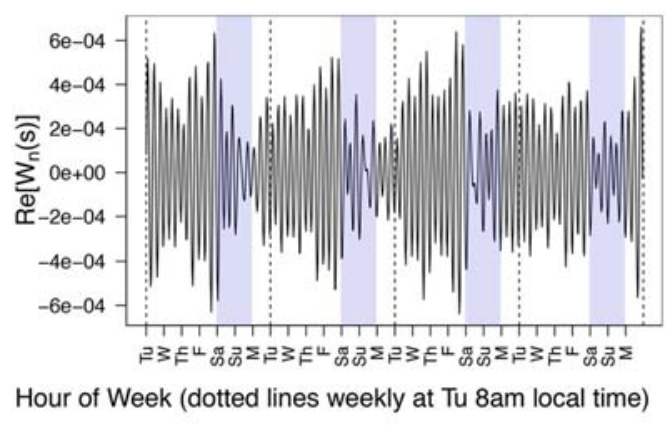

(b)

Fig. 4. (a) Amplitude components of the FFT for June and September month long alcohol time series. This analysis reveals that the weekly and daily frequency components dominate in both months. (b) Real component of wavelet amplitude over the month of June. Saturdays and Sundays are shaded.

\subsection{Continuous Wavelet Transform}

In addition to resolving periodic components of the behavior time series through Fourier transform (we found daily and weekly), we can assess if there are other frequency components in the time series that are not consistent through time (such as the 8-hour period described in the Time Series section above). Time-frequency domain approaches such as the continuous wavelet transform (CWT) allow us to capture these inconsistencies. A wavelet transform compares a signal with shifted and scaled copies of a basic wavelet. The CWT discretizes scale more finely than the discrete wavelet transform, allowing more flexibility to uncover underlying frequency components, which can be helpful in high-resolution applications such as this.

We used a Morlet wavelet, which is a complex-valued wavelet, composed of a complex exponential (carrier) multiplied by a Gaussian window (envelope). The Morlet wavelet has been used in population-epidemiological studies, assessing epidemic waves [45] and in high-frequency applications [30], given that it is very similar to an impulse component, along with its simplicity. It is also less influenced by nearby frequencies given that the Morlet wavelet, $\psi_{0}$, is a damped complex exponential. The continuous wavelet transform $(\mathrm{CWT}), W_{n}(s)$, of the time series is calculated as the convolution of $\mathbf{x}$ with a scaled and translated version of $\psi_{0}$ [84]. Figure $4 \mathrm{~b}$ shows the average amplitude of the real component of $W_{n}$ for the alcohol time series over all 28 days of June using a wavelet of 8-hour period.

\subsection{Similarity Measures}

To assess similarity between time series features, there are a variety of measures which are typically used on an application-dependent basis. Overall the goal is to compare and identify related temporal patterns, beyond the point values in each time series. Several similarity measures have been proposed for 1D and 2D signals. Here we chose the Euclidean distance to compare amplitudes of the time series of the behavior discussion, because (a) it has been shown useful in cases with similar dimensionality, and (b) it can be used with any other type of similarity measure, as long as this measure can be expressed as the Euclidean distance between feature vectors in some feature space. 
To compare phase distance, $d$ between series $t_{j}$ and $t_{k}$ we use:

$$
d\left(t_{j}, t_{k}\right)=\left|\operatorname{Arg}\left(\frac{T_{j}(f)}{T_{k}(f)}\right)\right|
$$

where $T$ is the Fourier transformed series and $f$ is the frequency of interest.

\subsection{Clustering}

After assessing similarity between the time series features, we use agglomerative hierarchical clustering to group and differentiate the temporal patterns of consumption posts. Dendrograms are a common method in time series data mining to find natural groupings of time series, due to the high dimensionality, potentially high feature correlation, and potentially large amounts of noise that characterize time series. A dendrogram is also attractive because it generates a clustering of $M$ objects and summarizes $O(M)$ measurements.

As a hierarchical method, a dendrogram is formed by finding successive clusters using previously established ones. Agglomerative hierarchical clustering algorithms begin with each element as a separate cluster and merge them into successively larger clusters. The merges and splits are determined in a greedy manner. The result is a dendrogram which looks like a tree, with branches for each component (time series). The height of a branch represents the mean distance between the clusters, illustrating which objects (days or weeks) are the most similar or different. So, branches that are closer at the leaves of the tree are from time series that are more similar. While comparing point values would describe the difference in exact amount of posting at a specific time, distance and clustering methods allow us to compare overall temporal features of behavior, which can be used in modeling efforts, help us better understand mechanisms behind behavior and shape intervention and prevention efforts. Further, this algorithm can tolerate noise that grows with the number of data points while still recovering the hierarchical clusters with high probability [10].

We also assessed the stability of these results via Baker's Gamma correlation coefficient, which is a measure of association (similarity) between two trees of hierarchical clustering (dendrograms) [9]. Baker's Gamma is defined as the rank correlation between the stages at which pairs of objects combine in each of the two trees. The value can range between -1 and 1 , with values near zero indicating that the two trees are not statistically similar. Using the alcohol dendrograms from daily time series (Figure 8), Baker's correlation was calculated successively by increasing the amount of sampled data (from $11 \%$ to $100 \%$ ). At each sampling rate we generated 100 pairs of dendrograms using sampling with replacement, and calculated the average Baker correlation of the pairs (Figure 9. All dendrograms in this work were generated using the cluster package in R. Branch colors were generated automatically based on resulting clusters using the factoextra package.

\section{RESULTS}

Overall, the goals of the above work were to 1) create time series of self-reported behavior communications about alcohol and tobacco consumption 2) assess temporal, periodic and phase representations of the data from (1) and 3) investigate if differences in group (age of posters about alcohol consumption) or tobacco product use could be identified using representations from (2) and time series clustering methods.

\subsection{Comparison to Other Time Series}

First, we compared the Twitter behavior time series to other data at similar temporal resolution. We found good correlation between the Twitter first-person behaviors and Google Trends, for alcohol, tobacco (non-hookah) and hookah (average pearson's correlation $\rho=0.72-0.88$, Table 1). Correlation with the Venmo data was not as strong $\rho=0.50$ and 0.44 for alcohol and hookah 


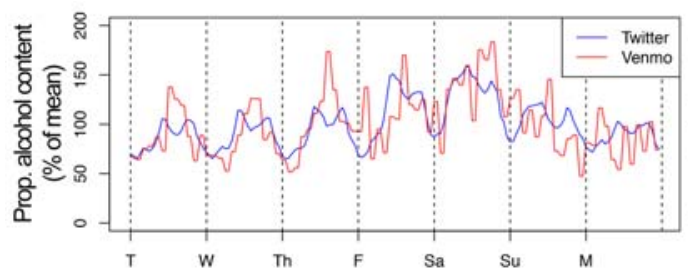

(a) Hour of Week

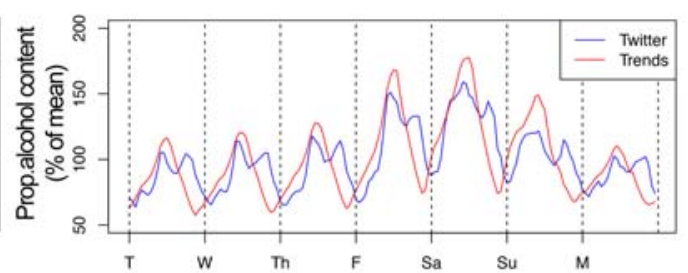

(b) Hour of Week

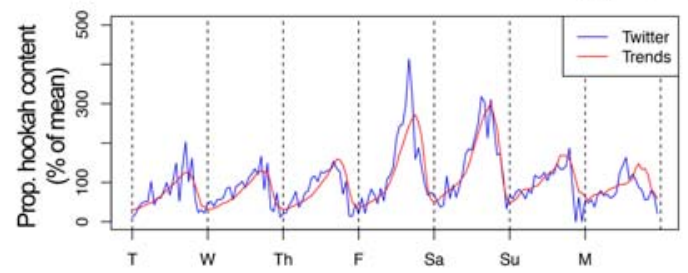

(c) Hour of Week

Fig. 5. Comparison of Twitter self-reports with (a) Venmo payments for alcohol (b) Google Trends for alcohol topics and (c) Google Trends for hookah topics. Dotted lines are at 8am. In (a) the Venmo data is at two-hour resolution for clarity. Venmo data is normalized by all transactions each hour, Google Trends are scaled based on proportion to all searches on all topics, and Twitter posts normalized to all Tweets from the API.

respectively). However these correlations are higher than if the Twitter data, for, say alcohol, was correlated with hookah transactions (last row on Table 1). There was not enough Venmo tobacco (excluding hookah) data for comparison - in total, 7.81\% users from the four weeks of Venmo data made alcohol-related transactions (identified through our selection criteria), while $0.10 \%$ made tobacco-related transactions.

However, the correlation values do not fully describe the similarities between the time series. The peak days for each data set and behavior align (Friday and Saturday for alcohol, Saturday and Friday for hookah, in that order). Further, as observed above, the Twitter alcohol data has two peaks, one at approximately 8pm and a smaller one a few hours later, while the Venmo alcohol data starts to increase $\sim 1$ hour after the initial Twitter peak (Figure $5 \mathrm{a}$ ). Similarly, on Friday and Saturday nights, the Venmo alcohol data starts to increase just after the Twitter data peaks. As Venmo represents people making payments to each other, it follows that we would not expect this data stream to correlate exactly with the Twitter self-reported behaviors, and it makes some intuitive sense that the spike in payments would follow the spike in consumption with some delay. These data sources must be compared while remaining cognizant that the data sources represent different different aspects of the behaviors (sharing, searching, payment). Payments from Venmo only represent a subset of drinking/smoking activities (namely, the drinking and smoking activities where friends are splitting a bill), while the Twitter data includes self-reports from all types of locations including those outside of bars and restaurants that do not involve payments and sharing of bills. Overall, we find the Venmo data useful for comparing at hourly and daily resolution. The fact that the Venmo alcohol time series trails Twitter's time series ( 0.62 correlation at a 2-hour lag, when both are taken at a 2-hour resolution), matches the intuition that payment and bill-splitting activity will lag behind consumption activity.

Though the Google time series increases at the same times as the Twitter alcohol and hookah series, the Google data does not exhibit the multiple peaks seen daily for the Twitter alcohol time series. The Google and Twitter peaks align around 7-8pm on weekdays and 9pm on weekends for 
alcohol, and between 1 and 3 am for hookah, depending on the day. The Google Trends alignment with the earlier Twitter peak for alcohol may result in the larger correlation with a three hour lag (Table 1), which is when the second Twitter peak starts to decrease (and the Google Trends peak already decreased about three hours earlier). We found that in general Google Trends for alcohol peaked slightly earlier than Twitter self-reports; a possible explanation, though it cannot be tested without surveys, is that some users will search for relevant keywords as a way of choosing venues to go to. For hookah, the Google and Twitter trends may align better as the Twitter hookah data peaks over a narrower time period during the day.

Overall, though each data source may be generated by completely different populations, the comparable temporal resolution enables us to assess the trends generally. We find that the Venmo transaction and Google Trends time series generally correspond with those from the Twitter reports of people engaging in the examined behaviors. As well, the comparisons help us elucidate that transaction or online search trends simply represent proxies about behavior, and further highlight the need to explore parsed reports about actual engagement in behaviors such as we have done from Twitter.

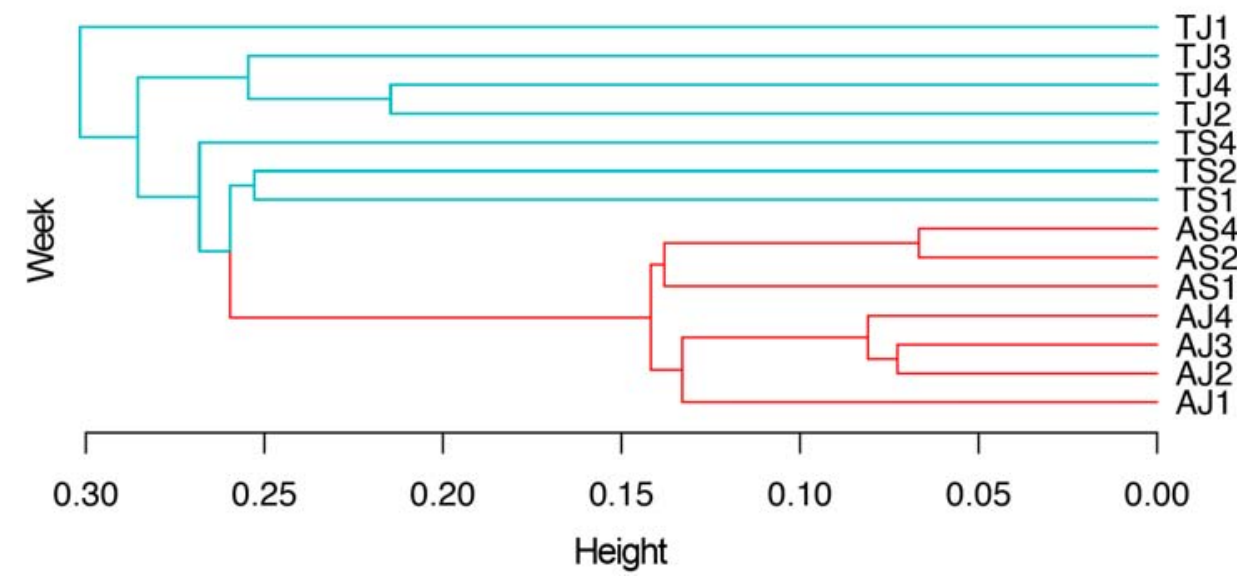

Fig. 6. Dendrogram illustrating differences in alcohol and tobacco time series. Time series for Tobacco are labelled starting with a $\mathrm{T}$, for alcohol with an A. Weeks with missing data are omitted.

\begin{tabular}{cc|cc}
\hline Data Set 1 & Data Set 2 & Correlation (lag 0) & Max correlation (lag) \\
\hline Twitter (alcohol) & Google (alcohol) & 0.72 & $0.81(+3)$ \\
Twitter (tobacco) & Google (tobacco) & 0.77 & $0.80(-1)$ \\
Twitter (hookah) & Google (hookah) & 0.88 & $0.90(-1)$ \\
Twitter (alcohol) & Venmo (alcohol) & 0.50 & $0.52(-1)$ \\
Twitter (hookah) & Venmo (hookah) & 0.44 & $0.52(+1)$ \\
\hline \hline Twitter (alcohol) & Venmo (hookah) & 0.16 & \\
\hline
\end{tabular}

Table 1. Correlation between time series. Bottom row included to show that although the Twitter/Venmo correlation not as good as Twitter/Google, it is still much better than off-topic correlations. 


\subsection{Alcohol and Tobacco Time Series Properties: Hourly and Day of the Week Trends}

This work goes beyond the resolution of survey based measures of weekly and daily trends of alcohol consumption $[5,42]$ and examines trends about alcohol consumption, through social media communications, at the sub-daily level using amplitude, frequency and phase.

5.2.1 Amplitude. Compared to tobacco-consumption posts, alcohol posting trends differ more from the overall Tweeting trends, having a larger evening spike (Figure 1). On the other hand, tobacco related posts were less different than overall Tweeting trends, still starting lower in the morning but not peaking as much later. This can be perhaps related to more consistent tobacco consumption patterns (people might smoke all the time versus drink more at certain times/days). While some of these differences are apparent visually, we also clearly show that the tobacco and alcohol time series differ by using Euclidean distance and clustering; all alcohol weeks cluster together and differently than the tobacco weeks (Figure 6).

Examining the alcohol time series on its own in-depth via clustering, we found that the time series amplitude for days of the week in June resolve differently Figure 7a. Weekend days (Saturday and Sunday) have different temporal patterns, but are closest to each other. Days early in the week (Monday and Tuesday) are also closer to each other compared to days later in the week (Wednesday, Thursday and Friday). While this result matches what one would expect, it has been shown quantitatively only once before, from data in one location: a study from the 1970's of drinkers in Boston [5]. Overall results showed temporal patterns of weekends/holidays to be most similar (closest together in Figure 8). Notably, one of the Mondays from the 8 weeks also maps closest to the Sundays, and this Monday is Labor day (in the first week of September, S1). Examining the time series for that Monday shows it follows more closely to a typical Sunday trend of a decreased evening proportion of alcohol-consumption Tweets. This difference is an example of the utility of the dendrogram; the difference is barely visible in the time series, but the day clearly resolves closer to Sundays in the dendrogram. We found all dendrogram results to be very stable (Figure 9), starting with sampling $11 \%$ of the data.

5.2.2 Phase. When comparing phase dissimilarity of the dominant frequency component (corresponding to a 24 hour period) of the daily time series, the dendrogram (Figure $7 \mathrm{~b}$ ) shows that Saturdays and Sundays have different phase from the weekdays, with the exception of the Labor Day Monday. This finding again confirms what we anecdotally would expect but provides novel empirical evidence; the time at which drinking posts start to increase by day differs with an earlier increase on weekends.

5.2.3 Frequency. The alcohol-consumption posts also show clear diurnal and daily trends in the time series and hourly, daily, weekly and monthly frequency components (Figures 1 and 4). The daily periodicity has a larger magnitude on weekends, a decreased 8-hour frequency component, and an earlier increase on weekends. Results from wavelet analysis indicated that the 8-hour component is decreased on weekends (decreased amplitude) which corroborates the visible 8-hour period/double peak on weekdays that is not visible on weekends (Figure 1a). Other wavelets such as a 24 hour-period, revealed differences across the days that are already visible in the time series. Further, the complex portion of the wavelet did not provide any added information indicating that there is no significant phase shift of the 8-hour component across days in the week.

\subsection{Time Series Comparisons Across Different Weeks}

Our work shows that by using social media data, we can also examine weekly trends at different times of year, whereas existing surveys have generally only been deployed once annually and thus results may not capture larger seasonal trends. Comparing the amplitude of every day separately 


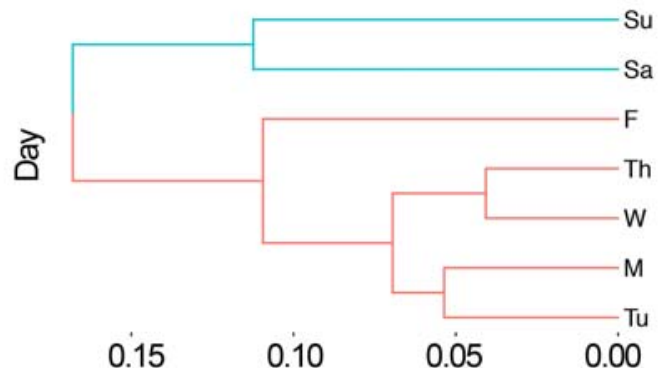

(a) Euclidean distance, alcohol
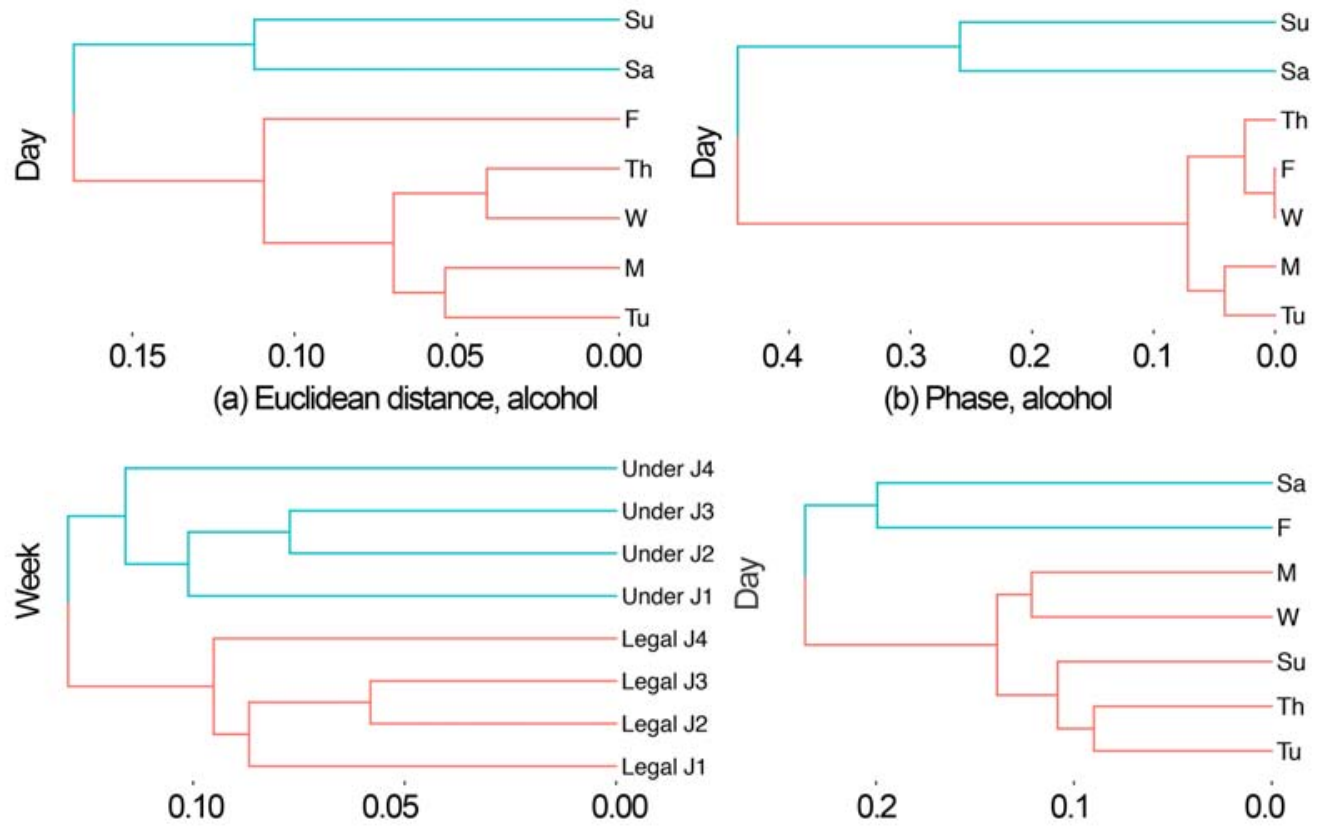

(c) Legal and Underage (June)

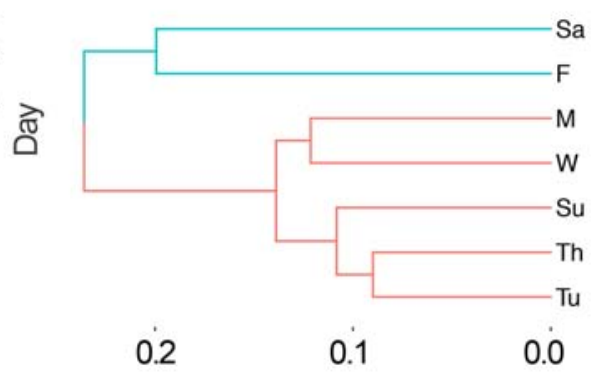

(d) Euclidean distance, hookah/all tobacco

Fig. 7. Dendrogram illustrating differences in (a) euclidean distance of amplitude of daily alcohol drinking post time series averaged over all weeks in June and September (b) difference in phase over all days in June and September (c) amplitude of weekly time series for legal and under age users in June and (d) Euclidean distance of amplitude of hookah-use posts compared to all tobacco-use posts in June and September.

for the alcohol-consumption posts (Figure 8) instead of averaging over all weeks shows that, of all the days across the two months, days early in the week (Monday through Thursday) are most similar. The figure also shows that Fridays are similar to each other through all weeks, and that the same is true for all Saturdays and Sundays. The labor day Monday also shows results similar to the preceding Sunday. And even for different months, these patterns by day of the week hold.

Finally in regards to distinguishing time series from different groups or about specific tobacco products, we see that inferred legal age and under age patterns of alcohol consumption posts weekly representations are dissimilar (Figure 7c), with time series from each group more similar to each other. As well, it is possible to distinguish temporal representations. Relative to all other tobacco consumption posts, hookah consumption posts show day-to-day patterns most similar on Friday and Saturday compared to other days of the week, over all weeks in June and September (Figure 7d). We discuss implications of these findings in the next section.

\section{DISCUSSION AND CONCLUSION}

We examined time and frequency-domain representations of social media communications where we applied natural language processing and machine learning methods to specifically identify self-reports about alcohol and tobacco consumption at sub-daily, daily and weekly resolutions. Our results go beyond existing survey-based studies finding that not only do overall consumption reports differ by day of week, but trends of consumption reports differ on weekends versus weekdays (starting earlier on weekends and having more distinct biphasic patterns on weekdays). We also 
show that trends of reports are similar by week at different times of the year. Finally through these methods we also show that posts about alcohol consumption behavior by inferred underage and legal age groups are dissimilar (consistently, week to week). Further, we find that posts about hookah consumption were more common on certain days (Friday and Saturday) compared to all tobacco consumption posts.

Though future work should entail a systematic validation study linking online reports to offline consumption behavior, we are confident in these findings as they confirm what has been found in isolated, low-resolution studies, through anecdotal sociological knowledge, and via correlation analysis with multiple proxy measures for alcohol and tobacco use (online Google searches and person-to-person Venmo payments). Further, this work demonstrates the added value of social media above survey measures for understanding behavioral patterns at scale. The comparison to other proxy measures reiterated the need for better measures of actual behavior; while online searches

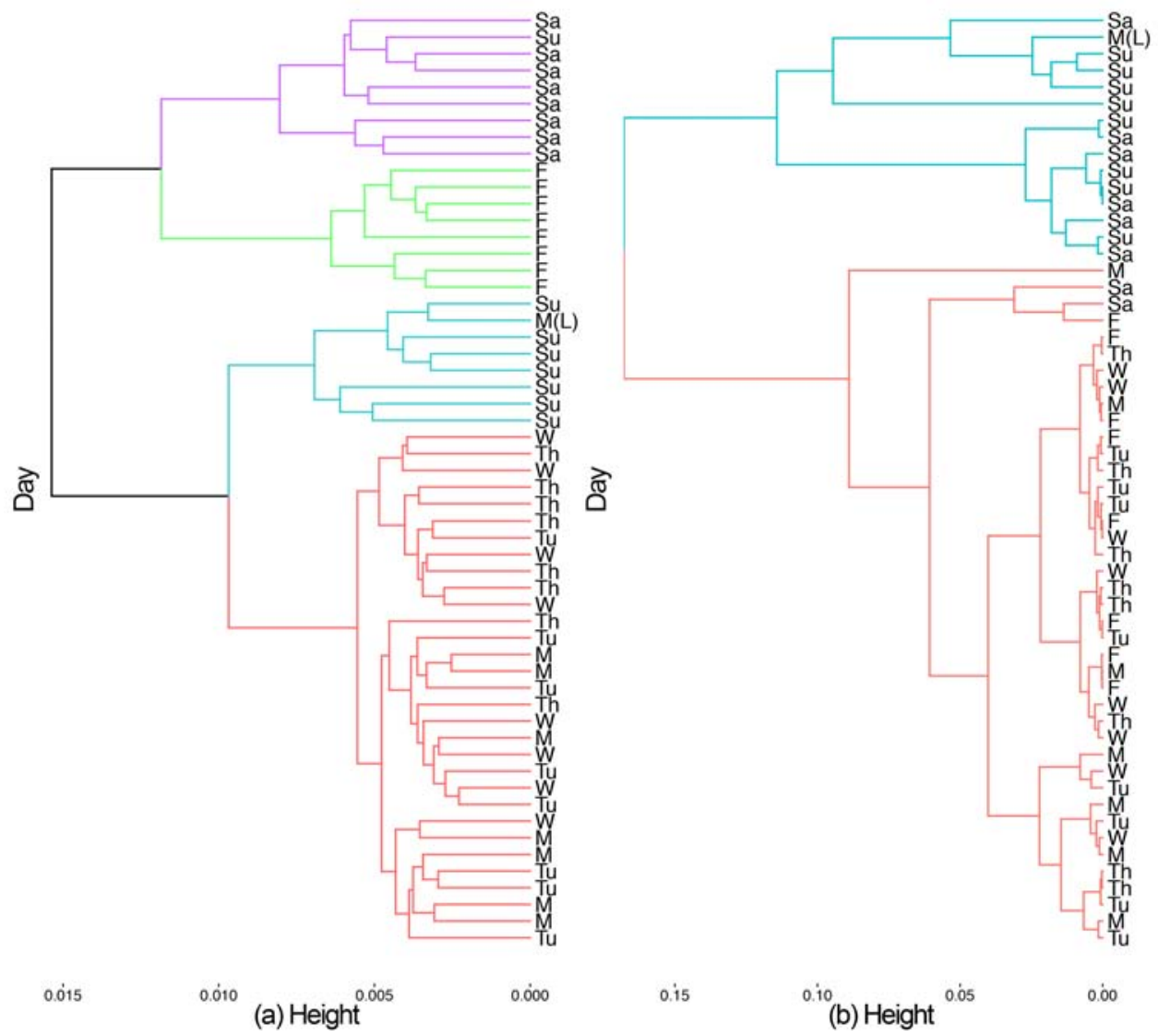

Fig. 8. (a) Dendrogram illustrating differences (using Euclidean distance) between amplitude of proportion alcohol drinking post time series. Labor day Monday, $M(L)$, resolves closer to the temporal patterns of alcohol communications on typical Sundays. (b) Dendrogram illustrating differences in phase of the first frequency component (24 hour) of daily alcohol drinking post time series. All days in June and September represented in both plots. 

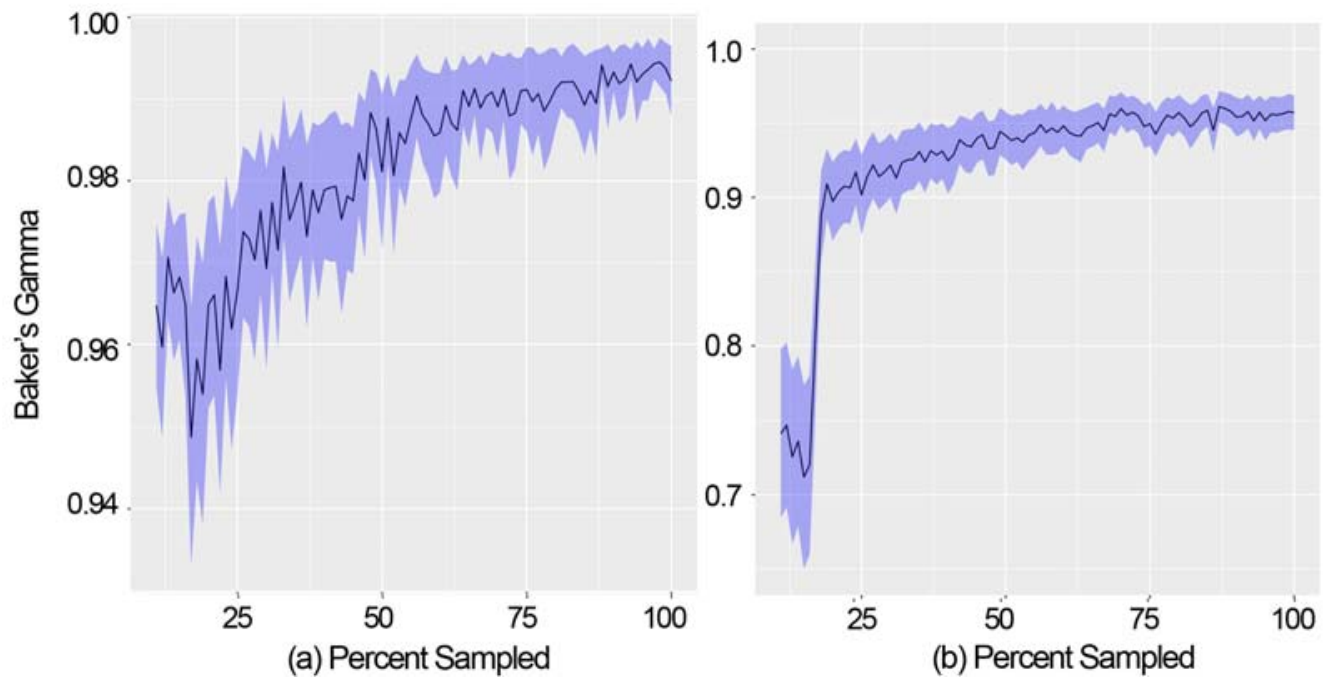

Fig. 9. For samples from 11 to $100 \%$ of the data (sampled with replacement), average Baker's Gamma correlation for each pair of resulting dendrograms from daily alcohol drinking time series using (a) Euclidean distance and (b) phase. 95\% confidence intervals shaded.

and payment trends generally correlated to daily alcohol and hookah self-reports (with expected lags), these are proxy measures that don't necessarily encompass behaviors as comprehensively (outside of payment or search-related situations) nor offer the same ability for deeper examination of behaviors such as through sentiment or subgroup/product analysis. The way behaviors are self-reported is itself also vital to take into account in a holistic socio-ecological framework that considers health communication, as well as the impact of communications on health outcomes.

\subsection{Findings in Relation to Alcohol and Tobacco Use}

Specifically for alcohol, we show how social media posts about consumption quantitatively differ during days and across days in the week and month. As well, overall weekly patterns of inferred underage and legal age people discussing alcohol consumption differ (whereas they may not on a daily or hourly basis). Also, social media discussion of hookah use differ by day, with Friday and Saturday being the highest relative to other tobacco products. In general, the ascertained temporal and frequency patterns can be used to shape sampling efforts for social media data going forward. For example, we did not find that days from June and September consistently clustered separately indicating that outside of specific anomalies (like the September Labor day), data from one month may serve as a similar cross-section to other months (at the monthly level). Temporal patterns from days of the week (e.g. Monday versus Saturday) consistently cluster separately, so data samples from different days of the week should be noted. Methods here can also reveal more nuanced features of the discussion of behaviors, such as how the time of increase of alcohol posts during the day is earlier on weekends compared to weekdays.

\subsection{Limitations}

Although this work provides a novel approach that can be used to improve understanding of behaviors, we acknowledge limitations of the work. First, in evaluating the time series of social media posts related to different types of behaviors (alcohol and tobacco consumption), we found 
that there are limits to the amount of data about a behavior that is required to understand and distinguish temporal representations. This limitation can be attributed to several components: 1) amount of posts about the topic on the social media platform, 2) performance of the classifier, 3) variation in the topic by day, 4) limitations of differences detectable by clustering techniques.

Specifically here, self-report biases can also affect the identified temporal patterns. For example, those who self-report age on Twitter may be only a certain type of people, and this group may not overlap with those who self-report substance use. Or, the temporal patterns of those who self-report on Twitter may not be representative of the entire population. It should be noted that the reliability, validity and stability of self-report measures are of great importance especially with regards to substance use. Even with other (survey) modalities of data collection, there are concerns such as age-biases in how substance use is reported through surveys [18, 76]. This is a fundamental limitation of this study in terms of the content of the data; we have not linked the Tweets to who people are or what people do in the offline world, thus all findings are based on how these behaviors are communicated online. Initial work assessing online and offline behavior has shown that reliable data about political behavior (candidates supported online and voting behavior) [32], and weight loss [65] are also validated in the offline world. As we discuss below, a comprehensive study that links the social media communications to offline behaviors should be performed.

Another significant limitation is representativeness of the data. Representativeness is one reason we demonstrated one measurement of group differences in the self-report of alcohol use (e.g. by age). Figures 3 and 7c (under/over age time series and dendrogram) show that temporal trends, while overall have some similarities, can still be differentiated via dendrogram clustering. In other words, the overall trends reported from social media should not be assumed to represent the entire population; if the results were stratified by group we will likely observe group-specific patterns Thus, we should make efforts to study what groups the data does represent. For other linked demographic or group information, while there are inference techniques we can use to assess other demographics besides age, this is a fundamental limitation. It must be carefully considered how to use findings from analysis of this data; instead of supplanting representative survey-based measures, the high-frequency and other high-resolution aspects of the data can potentially be harnessed to generate hypotheses, investigate sub-groups and inform offline studies.

Besides addressing online versus offline behaviors, epidemiological studies to compute incidence, health-related factors or outcomes are generally evaluated via concurrent validity. That is, comparing the data over time against another related standard. Though we showed some parallels to other highly used data sources such as Venmo purchases and Google Trends here (Google is the most popular search engine in the United States [34], and the total number of Venmo users in our 4 weeks of data was $8,973,726$ ). We believe that ground truth data about these behaviors at the desired time scale does not exist at the moment and social media reports can contribute towards this gap.

\subsection{Future Work}

A natural extension of the work would be to perform a study to evaluate how high-resolution communication of behaviors online compares to offline behaviors. This would involve a systematic study that examines the relationship between social media communications and offline behaviors for different groups, times and locations. This is an important next step for this work, to show which groups are being represented and how the reported behaviors correspond to real-world behavior. Social media data and the approach presented here can also inform future survey-based research to learn more about causes and mechanisms of identified patterns, and for further nuanced examination (e.g. specifically evaluating spring break behavior of underage individuals). In the 
future, identified representations of behavior can also be integrated into models to predict and identify time periods at which there are behavioral anomalies.

Future work can also be focused on extending the presented pipeline (natural language processing from social media and time-series analyses) to reports of other behaviors such as engaging in physical activity or eating healthy/unhealthy foods - which have obvious intervention possibilities if linked to time and place and also are commonly reported on social media [1]. Based on our findings, we found behaviors that tend to be more social or public discussed more heavily on social media (e.g. hookah versus cigarettes). Another natural extension of this work should examine the theoretical limits of the time series (e.g. how much behavioral communication, what is the minimum proportion of Tweets on a specific topic required to generate and differentiate the daily or weekly features). While there is some research on computing the limits of clustering techniques, these studies have not taken into account data from classified social media text [54].

\subsection{Behavioral and Precision Medicine Implications}

There are multiple key implications of this work. First, this work can potentially help enable a better understanding behaviors and their patterns than is currently available. Until now, our only understanding of behavior comes from low-frequency surveys which suffer from recall and information biases. High-resolution communications about behaviors can thus help fundamentally advance behavioral theories by improving our understanding of the mechanisms behind behavior changes and choices that happen every day. The behavioral science community has identified that more real-time data sources are needed to better understand how and why people make decisions especially related to health $[64,80]$. High-resolution behavior communications can could potentially be linked to appropriate representations of off-line behavior (e.g. at the right temporal resolution, or amplitude or phase components) and used to include behavioral features in individual and population-level prediction models.

While the methods as described use population-level behavior communications, with enough data, one can also learn individual-specific representations of behavior. However, populationrepresentations of behavior are also important for shaping policies (as articulated below), and have been used to asses health-related phenomena in areas such as air pollution and mortality, food perception and health expressions [28, 31, 60]. Further, even in study of individual health trajectories, concurrent population behaviors can improve prediction [74] as has been done in studies using physiological data. This again signals the need to know how to appropriately represent population-level behaviors and their communications.

\subsection{Public Health Implications}

In public health, high-resolution measures of behaviors or communications about them also have many applications. For example in this work we found that inferred legal age drinkers communicate about consumption earlier on Saturdays than under age individuals. Such findings can have direct implications for the design and implementation of focused public health policies such as extra vigilance on identification checks at times and places where underage consumption is highest. There have been many examples of public healthy policies shaped by research on risky behaviors and locations. Those examples include increased taxation on sugary drinks as well as closing hours for bars, and restricting sales at certain times [35, 47, 49, 58].

\subsection{Implications for the Design of Social Computing Systems}

High-resolution behavior measures, once-linked, or high-resolution communications about behaviors can also eventually be used towards development of social computing systems. Communication about behaviors can help inform the focus of studies and interventions by ascertaining which 
precise spatial and temporal circumstances are linked to specific behaviors. For example, should we compute tobacco consumption levels over the course of a day or a week before intervening? Are there specific times to that particular groups discuss consumption versus others? Answers to these questions are increasingly important amidst growing evidence supporting the development of real or "just-in-time" interventions. Overall these studies will better inform intervention and prevention efforts by improving effectiveness and optimizing investments in public health endeavors.

\section{ACKNOWLEDGMENTS}

The authors acknowledge support by the National Institutes of Health, National Institute of Alcohol Abuse and Alcoholism and National Institute of Drug Abuse (NIAAA, NIDA) under grants R21 AA023901 and R21 MH110190, and a grant from the Truth Initiative.

\section{APPENDIX}

\subsection{Twitter and Venmo keywords used for hookah filtering}

hooka, hookas, hookah, shisha, shishas, sheesha, sheeshas, narghile, narghiles, arghileh, arghilehs, hubble bubble, hubblebubble, hubbles bubble, hubbles bubbles, hubble bubbles

\subsection{Twitter and Venmo keywords used for tobacco (non-hookah) filtering}

tobacco, smoke, smoking, cigg, cigaret, cigar, cancerette, loosys, coffin nail, cancer sticks, zig zaggs, zig-zag, marlboro, benson hedges, pall mall, nicotin, camel, nweport, rothmans, e-cig, ecig, electronic cig, vape, vaping, e-liquid, ejuice, esmoke, eliquid, e-juice, e-smoke, juul, vaporfi, vype pebble, v2cigs, halocigs, krave, as well as the emoji (Venmo only):

\begin{tabular}{|c|c|c|c|c|}
\hline Class Label & Positive & $\beta$ & Negative & $\bar{\beta}$ \\
\hline \multirow[t]{5}{*}{ alcohol } & drunk & 52.44 & coffee & -13.60 \\
\hline & beer & 42.24 & water & -13.59 \\
\hline & drinking & 36.21 & tea & -11.65 \\
\hline & wine & 36.21 & soda & -10.44 \\
\hline & drink & 25.02 & drunk no & -9.7 \\
\hline \multirow[t]{5}{*}{ first person | alcohol } & $\mathrm{i}$ & 16.55 & you & -10.71 \\
\hline & drinking & 16.10 & she & -10.10 \\
\hline & i'm & 12.70 & he & -10.04 \\
\hline & drunk & 10.04 & people & -9.88 \\
\hline & with my & 9.08 & they & -9.45 \\
\hline \multirow[t]{5}{*}{ present | first person | alcohol } & drinking a & 12.12 & tonight & -10.57 \\
\hline & drunk & 10.55 & get drunk & -10.09 \\
\hline & last drink & 7.59 & was & -9.25 \\
\hline & I'm drunk & 5.69 & wanna & -9.00 \\
\hline & drink drink & 5.84 & who & 8.96 \\
\hline \multirow[t]{5}{*}{ tobacco } & cigarettes & 48.46 & company & -19.02 \\
\hline & vaping & 46.23 & kong & -11.23 \\
\hline & cigarette & 44.38 & time to smoke & -10.29 \\
\hline & tobacco & 38.26 & pipes & -10.19 \\
\hline & cigs & 37.27 & weed & -10.16 \\
\hline \multirow[t]{5}{*}{ first person $\mid$ tobacco } & i & 20.98 & is & -7.84 \\
\hline & my & 18.67 & you & -7.79 \\
\hline & i'm & 16.13 & your & -6.98 \\
\hline & time & 12.14 & tobacco & -6.34 \\
\hline & me & 11.28 & people & -5.87 \\
\hline \multirow[t]{5}{*}{ present $\mid$ first person $\mid$ tobacco } & smoking & 16.98 & smoked & -8.94 \\
\hline & i smoke & 8.37 & $\mathrm{i}$ & -7.29 \\
\hline & vaping & 6.97 & a & -7.24 \\
\hline & smoking a & 6.77 & to & -7.17 \\
\hline & love & 6.10 & done smoking & -7.12 \\
\hline
\end{tabular}

Table 2. Top features (no expression boundaries) for each classification stage and their associated feature strength, $\beta$ (weighted via inverse document frequency). 


\subsection{Venmo alcohol keywords and emojis}

Keywords: drink, drank, wine, champagne, alcohol, beer, bottle, cocktail, bar, brewery, lounge, pub, liquor, booze, vodka, tequila, gin, ciroc, margarita, shot, whiskey, lager, tipsy, drunk, sober, wasted, pregame, pregaming.

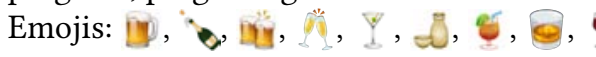

\section{REFERENCES}

[1] Sofiane Abbar, Yelena Mejova, and Ingmar Weber. 2015. You tweet what you eat: Studying food consumption through twitter. In Proceedings of the 33rd Annual ACM Conference on Human Factors in Computing Systems. ACM, 3197-3206.

[2] Faiyaz Al Zamal, Wendy Liu, and Derek Ruths. 2012. Homophily and Latent Attribute Inference: Inferring Latent Attributes of Twitter Users from Neighbors. ICWSM 270 (2012).

[3] Cláudia M Antunes and Arlindo L Oliveira. 2001. Temporal data mining: An overview. In KDD workshop on temporal data mining, Vol. 1. 13.

[4] Milton Argeriou. 1975. Daily alcohol consumption patterns in Boston: some findings and a partial test of the Tuesday hypothesis. Journal of studies on alcohol 36, 11 (1975), 1578-1583.

[5] Milton Argeriou. 1975. Daily alcohol consumption patterns in Boston: some findings and a partial test of the Tuesday hypothesis. Journal of studies on alcohol 36, 11 (1975), 1578-1583.

[6] John W Ayers, Benjamin M Althouse, Jon-Patrick Allem, J Niels Rosenquist, and Daniel E Ford. 2013. Seasonality in seeking mental health information on Google. American journal of preventive medicine 44, 5 (2013), 520-525.

[7] John W Ayers, Eric C Leas, Jon-Patrick Allem, Adrian Benton, Mark Dredze, Benjamin M Althouse, Tess B Cruz, and Jennifer B Unger. 2017. Why do people use electronic nicotine delivery systems (electronic cigarettes)? A content analysis of Twitter, 2012-2015. PloS one 12, 3 (2017), e0170702.

[8] Sangwon Bae, Denzil Ferreria, Brian Suffoletto, Juan C Puyana, Ryan Kurtz, Tammy Chung, and Anind K Dey. 2017. Detecting Drinking Episodes in Young Adults Using Smartphone-based Sensors. (2017).

[9] Frank B Baker. 1974. Stability of two hierarchical grouping techniques Case I: Sensitivity to data errors. F. Amer. Statist. Assoc. 69, 346 (1974), 440-445.

[10] Sivaraman Balakrishnan, Min Xu, Akshay Krishnamurthy, and Aarti Singh. 2011. Noise thresholds for spectral clustering. In Advances in Neural Information Processing Systems. 954-962.

[11] Han Bantje. 1987. Seasonality of births and birthweights in Tanzania. Social Science Medicine 24, 9 (1987), $733-739$.

[12] Jakob Bardram. 1998. Designing for the dynamics of cooperative work activities. In Proceedings of the 1998 ACM conference on Computer supported cooperative work. ACM, 89-98.

[13] Jakob E Bardram. 2000. Temporal coordination-on time and coordination of collaborativeactivities at a surgical department. Computer Supported Cooperative Work (CSCW) 9, 2 (2000), 157-187.

[14] Carla Alexia Campbell, Robert A Hahn, Randy Elder, Robert Brewer, Sajal Chattopadhyay, Jonathan Fielding, Timothy S Naimi, Traci Toomey, Briana Lawrence, Jennifer Cook Middleton, et al. 2009. The effectiveness of limiting alcohol outlet density as a means of reducing excessive alcohol consumption and alcohol-related harms. American journal of preventive medicine 37, 6 (2009), 556-569.

[15] Patricia A Cavazos-Rehg, Melissa Krauss, Sherri L Fisher, Patricia Salyer, Richard A Grucza, and Laura Jean Bierut. 2015. Twitter chatter about marijuana. Journal of Adolescent Health 56, 2 (2015), 139-145.

[16] Emily H Chan, Vikram Sahai, Corrie Conrad, and John S Brownstein. 2011. Using web search query data to monitor dengue epidemics: a new model for neglected tropical disease surveillance. PLoS Negl Trop Dis 5, 5 (2011), e1206.

[17] Stevie Chancellor, Zhiyuan Lin, Erica L Goodman, Stephanie Zerwas, and Munmun De Choudhury. 2016. Quantifying and Predicting Mental Illness Severity in Online Pro-Eating Disorder Communities. In Proceedings of the 19th ACM Conference on Computer-Supported Cooperative Work \& Social Computing. ACM, 1171-1184.

[18] Chuan-Yu Chen and James C Anthony. 2003. Possible age-associated bias in reporting of clinical features of drug dependence: epidemiological evidence on adolescent-onset marijuana use. Addiction 98, 1 (2003), 71-82.

[19] Yunan Chen. 2011. Health information use in chronic care cycles. In Proceedings of the ACM 2011 conference on Computer supported cooperative work. ACM, 485-488.

[20] Martin J Chorley, Luca Rossi, Gareth Tyson, Matthew J Williams, et al. 2016. Pub Crawling at Scale: Tapping Untappd to Explore Social Drinking.. In ICWSM. 62-71.

[21] Rumi Chunara, Jason Andrews, and John Brownstein. 2011. Social and News Media Enable Estimation of Epidemiological Patterns Early in the 2010 Haitian Cholera Outbreak. American fournal of Tropical Medicine and Hygiene 86 (2011), $39-45$.

[22] Rumi Chunara, Lauren E Wisk, and Elissa R Weitzman. 2017. Denominator Issues for Personally Generated Data in Population Health Monitoring. American fournal of Preventive Medicine 52, 4 (2017), 549-553. 
[23] Matthew Cochrane. 2017. Why Venmo Is So Popular With Millennials. https://www.fool.com/investing/2017/03/22/ why-venmo-is-so-popular-with-millennials.aspx. (2017). Accessed: 2017-07-06.

[24] Sunny Consolvo, David W McDonald, Tammy Toscos, Mike Y Chen, Jon Froehlich, Beverly Harrison, Predrag Klasnja, Anthony LaMarca, Louis LeGrand, Ryan Libby, et al. 2008. Activity sensing in the wild: a field trial of ubifit garden. In Proceedings of the SIGCHI Conference on Human Factors in Computing Systems. ACM, 1797-1806.

[25] SM Cook, RI Glass, CW LeBaron, and Mei-Shang Ho. 1990. Global seasonality of rotavirus infections. Bulletin of the World Health Organization 68, 2 (1990), 171.

[26] Munmun De Choudhury, Scott Counts, and Eric Horvitz. 2013. Social media as a measurement tool of depression in populations. In Proceedings of the 5th Annual ACM Web Science Conference. ACM, 47-56.

[27] Munmun De Choudhury, Emre Kiciman, Mark Dredze, Glen Coppersmith, and Mrinal Kumar. 2016. Discovering shifts to suicidal ideation from mental health content in social media. In Proceedings of the 2016 CHI Conference on Human Factors in Computing Systems. ACM, 2098-2110.

[28] Munmun De Choudhury, Sanket Sharma, Tomaz Logar, Wouter Eekhout, René Nielsen, Georgia Tech, and Global Pulse. 2016. Quantifying and Understanding Gender and Cross-Cultural Differences in Mental Health Expression via Social Media. (2016).

[29] Munmun De Choudhury, Sanket S Sharma, Tomaz Logar, Wouter Eekhout, and René Clausen Nielsen. 2017. Gender and Cross-Cultural Differences in Social Media Disclosures of Mental Illness. (2017).

[30] Hasan Demirel and Gholamreza Anbarjafari. 2010. Satellite image resolution enhancement using complex wavelet transform. IEEE geoscience and remote sensing letters 7, 1 (2010), 123-126.

[31] Qian Di, Yan Wang, Antonella Zanobetti, Yun Wang, Petros Koutrakis, Christine Choirat, Francesca Dominici, and Joel D Schwartz. 2017. Air Pollution and Mortality in the Medicare Population. New England fournal of Medicine 376 , 26 (2017), 2513-2522.

[32] Joseph DiGrazia, Karissa McKelvey, Johan Bollen, and Fabio Rojas. 2013. More tweets, more votes: Social media as a quantitative indicator of political behavior. PloS one 8, 11 (2013), e79449.

[33] Danice K Eaton, Laura Kann, Steve Kinchen, Shari Shanklin, James Ross, Joseph Hawkins, H Harris, R Lowry, T McManus, and D Chyen. 2011. Youth risk behavior surveillance-United States, 2009. Morbidity and Mortality Weekly Report 59 (2011).

[34] eBiz. 2017. Top 15 Most Popular Search Engines. http://www.ebizmba.com/articles/search-engines. (2017). Accessed 2017-07-08.

[35] Brian Elbel, Rogan Kersh, Victoria L Brescoll, and L Beth Dixon. 2009. Calorie labeling and food choices: a first look at the effects on low-income people in New York City. Health affairs 28, 6 (2009), w1110-w1121.

[36] Simon Foster, Gerhard Gmel, Natalia EstÃlvez, Caroline BÃdhler, and Meichun Mohler-Kuo. 2015. Temporal patterns of alcohol consumption and alcohol-related road accidents in young Swiss men: seasonal, weekday and public holiday effects. Alcohol and alcoholism 50, 5 (2015), 565-572. http://alcalc.oxfordjournals.org/content/50/5/565.long

[37] Adam Fourney, Ryen W White, and Eric Horvitz. 2015. Exploring time-dependent concerns about pregnancy and childbirth from search logs. In Proceedings of the 33rd Annual ACM Conference on Human Factors in Computing Systems. ACM, 737-746.

[38] Caroline Free, Rosemary Knight, Steven Robertson, Robyn Whittaker, Phil Edwards, Weiwei Zhou, Anthony Rodgers, John Cairns, Michael G Kenward, and Ian Roberts. 2011. Smoking cessation support delivered via mobile phone text messaging (txt2stop): a single-blind, randomised trial. The Lancet 378, 9785 (2011), 49-55.

[39] Venkata Rama Kiran Garimella, Abdulrahman Alfayad, and Ingmar Weber. 2016. Social media image analysis for public health. In Proceedings of the 2016 CHI Conference on Human Factors in Computing Systems. ACM, 5543-5547.

[40] Xianping Ge and Padhraic Smyth. 2000. Deformable Markov model templates for time-series pattern matching. In Proceedings of the sixth ACM SIGKDD international conference on Knowledge discovery and data mining. ACM, 81-90.

[41] Scott A Golder and Michael W Macy. 2011. Diurnal and seasonal mood vary with work, sleep, and daylength across diverse cultures. Science 333, 6051 (2011), 1878-1881.

[42] Mark S Goldman, Paul E Greenbaum, Jack Darkes, Karen Obremski Brandon, and Frances K Del Boca. 2011. How many versus how much: 52 weeks of alcohol consumption in emerging adults. Psychology of Addictive Behaviors 25, 1 (2011), 16 .

[43] Google. 2017. Google Trends. https://support.google.com/trends/answer/4359550?hl=en. (2017). Accessed: 2017-07-06

[44] Google. 2017. Google Trends API Layer. https://github.com/GeneralMills/pytrends. (2017). Accessed: 2017-07-06.

[45] Brian Grenfell, Ottar Bjørnstad, and J Kappey. 2001. Travelling waves and spatial hierarchies in measles epidemics. Nature 414, 6865 (2001), 716-723.

[46] Nir Grinberg, Mor Naaman, Blake Shaw, and Gilad Lotan. 2013. Extracting Diurnal Patterns of Real World Activity from Social Media.. In ICWSM.

[47] Jeffrey Grogger. 2017. Soda taxes and the prices of sodas and other drinks: evidence from Mexico. American fournal of Agricultural Economics 99, 2 (2017), 481-498. 
[48] Valery Guralnik and Jaideep Srivastava. 1999. Event detection from time series data. In Proceedings of the fifth ACM SIGKDD international conference on Knowledge discovery and data mining. ACM, 33-42.

[49] Robert A Hahn, Jennifer L Kuzara, Randy Elder, Robert Brewer, Sajal Chattopadhyay, Jonathan Fielding, Timothy S Naimi, Traci Toomey, Jennifer Cook Middleton, Briana Lawrence, et al. 2010. Effectiveness of policies restricting hours of alcohol sales in preventing excessive alcohol consumption and related harms. American journal of preventive medicine 39, 6 (2010), 590-604.

[50] Nabil Hossain, Tianran Hu, Roghayeh Feizi, Ann Marie White, Jiebo Luo, and Henry A Kautz. 2016. Precise Localization of Homes and Activities: Detecting Drinking-While-Tweeting Patterns in Communities.. In ICWSM. 587-590.

[51] Emre Kıcıman and Matthew Richardson. 2015. Towards decision support and goal achievement: Identifying actionoutcome relationships from social media. In Proceedings of the 21th ACM SIGKDD International Conference on Knowledge Discovery and Data Mining. ACM, 547-556.

[52] Santosh Kumar, Wendy Nilsen, Misha Pavel, and Mani Srivastava. 2013. Mobile health: Revolutionizing healthcare through transdisciplinary research. Computer 46, 1 (2013), 28-35.

[53] Jiaxin Liu, Elissa R Weitzman, and Rumi Chunara. 2017. Assessing Behavior Stage Progression From Social Media Data. In Proceedings of the 2017 ACM Conference on Computer Supported Cooperative Work and Social Computing. ACM, 1320-1333.

[54] Ulrike V Luxburg, Olivier Bousquet, and Mikhail Belkin. 2005. Limits of spectral clustering. In Advances in neural information processing systems. 857-864.

[55] Thomas W Mangione, Jonathan Howland, Benjamin Amick, Jennifer Cote, Marianne Lee, Nicole Bell, and Sol Levine. 1999. Employee drinking practices and work performance. fournal of studies on alcohol 60, 2 (1999), 261-270.

[56] Mary P Martinasek, Linda G Haddad, Christopher W Wheldon, and Tracey E Barnett. 2017. Beliefs and Attitudes Associated With Hookah Smoking Among a United States College Population. Respiratory Care 62, 3 (2017), 370-379.

[57] David J McIver, Jared B Hawkins, Rumi Chunara, Arnaub K Chatterjee, Aman Bhandari, Timothy P Fitzgerald, Sachin H Jain, and John S Brownstein. 2015. Characterizing sleep issues using twitter. Fournal of medical Internet research 17, 6 (2015).

[58] Jennifer Cook Middleton, Robert A Hahn, Jennifer L Kuzara, Randy Elder, Robert Brewer, Sajal Chattopadhyay, Jonathan Fielding, Timothy S Naimi, Traci Toomey, Briana Lawrence, et al. 2010. Effectiveness of policies maintaining or restricting days of alcohol sales on excessive alcohol consumption and related harms. American journal of preventive medicine 39, 6 (2010), 575-589.

[59] Mor Naaman, Jeffrey Boase, and Chih-Hui Lai. 2010. Is it really about me?: message content in social awareness streams. In Proceedings of the 2010 ACM conference on Computer supported cooperative work. ACM, 189-192.

[60] Ferda Ofli, Yusuf Aytar, Ingmar Weber, Raggi al Hammouri, and Antonio Torralba. 2017. Is Saki\# delicious?: The Food Perception Gap on Instagram and Its Relation to Health. In Proceedings of the 26th International Conference on World Wide Web. International World Wide Web Conferences Steering Committee, 509-518.

[61] Columbia University. National Center on Addiction and Substance Abuse. [n. d.]. The National Center on Addiction and Substance Abuse at Columbia University... Annual Report. National Center on Addiction and Substance Abuse at Columbia University

[62] Alan V Oppenheim and Ronald W Schafer. 2010. Discrete-time signal processing. Pearson Higher Education.

[63] Nicolas MF Oyane, Ingvar Bjelland, StÃěle Pallesen, Fred Holsten, and BjÃÿrn Bjorvatn. 2008. Seasonality is associated with anxiety and depression: the Hordaland health study. Fournal of Affective disorders 105, 1 (2008), 147-155.

[64] Gillian A OâÁŹReilly and Donna Spruijt-Metz. 2013. Current mHealth technologies for physical activity assessment and promotion. American journal of preventive medicine 45, 4 (2013), 501-507.

[65] Sherry Pagoto, Kristin L Schneider, Martinus Evans, Molly E Waring, Brad Appelhans, Andrew M Busch, Matthew C Whited, Herpreet Thind, and Michelle Ziedonis. 2014. Tweeting it off: characteristics of adults who tweet about a weight loss attempt. Journal of the American Medical Informatics Association 21, 6 (2014), 1032-1037.

[66] Fabian Pedregosa, Gaël Varoquaux, Alexandre Gramfort, Vincent Michel, Bertrand Thirion, Olivier Grisel, Mathieu Blondel, Peter Prettenhofer, Ron Weiss, Vincent Dubourg, et al. 2011. Scikit-learn: Machine learning in Python. fournal of Machine Learning Research 12, Oct (2011), 2825-2830.

[67] Pierre Philip, F Vervialle, P Le Breton, Jacques Taillard, and James A Horne. 2001. Fatigue, alcohol, and serious road crashes in France: factorial study of national data. Bmj 322, 7290 (2001), 829-830.

[68] Tobias Preis, Helen Susannah Moat, and H Eugene Stanley. 2013. Quantifying trading behavior in financial markets using Google Trends. Scientific reports 3 (2013), srep01684.

[69] Davide Proserpio, Scott Counts, and Apurv Jain. 2016. The psychology of job loss: using social media data to characterize and predict unemployment. In Proceedings of the 8th ACM Conference on Web Science. ACM, 223-232.

[70] Madhu Reddy and Paul Dourish. 2002. A finger on the pulse: temporal rhythms and information seeking in medical work. In Proceedings of the 2002 ACM conference on Computer supported cooperative work. ACM, 344-353. 
[71] William T Riley, Daniel E Rivera, Audie A Atienza, Wendy Nilsen, Susannah M Allison, and Robin Mermelstein. 2011. Health behavior models in the age of mobile interventions: are our theories up to the task? Translational behavioral medicine 1, 1 (2011), 53-71.

[72] Maria Roditis, Kevin Delucchi, David Cash, and Bonnie Halpern-Felsher. 2016. Adolescents' perceptions of health risks, social risks, and benefits differ across tobacco products. fournal of Adolescent Health 58, 5 (2016), 558-566.

[73] Marcel Salathe, Linus Bengtsson, Todd J Bodnar, Devon D Brewer, John S Brownstein, Caroline Buckee, Ellsworth M Campbell, Ciro Cattuto, Shashank Khandelwal, Patricia L Mabry, et al. 2012. Digital epidemiology. PLoS Comput Biol 8 , 7 (2012), e1002616.

[74] Peter Schulam and Suchi Saria. 2015. A framework for individualizing predictions of disease trajectories by exploiting multi-resolution structure. In Advances in Neural Information Processing Systems. 748-756.

[75] Ari Seifter, Alison Schwarzwalder, Kate Geis, and John Aucott. 2010. The utility of âĂIJGoogle TrendsâĂİ for epidemiological research: Lyme disease as an example. Geospatial health 4, 2 (2010), 135-137.

[76] Audrey M Shillington and JD Clapp. 2000. Self-report stability of adolescent substance use: are there differences for gender, ethnicity and age? Drug and alcohol dependence 60, 1 (2000), 19-27.

[77] Alessio Signorini, Alberto Maria Segre, and Philip M Polgreen. 2011. The use of Twitter to track levels of disease activity and public concern in the US during the influenza A H1N1 pandemic. PloS one 6, 5 (2011), e19467.

[78] Tushar Singh. 2016. Tobacco use among middle and high school studentsâĂŤUnited States, 2011-2015. MMWR. Morbidity and mortality weekly report 65 (2016).

[79] Luke Sloan, Jeffrey Morgan, Pete Burnap, and Matthew Williams. 2015. Who tweets? Deriving the demographic characteristics of age, occupation and social class from Twitter user meta-data. PloS one 10, 3 (2015), e0115545.

[80] Donna Spruijt-Metz, Eric Hekler, Niilo Saranummi, Stephen Intille, Ilkka Korhonen, Wendy Nilsen, Daniel E Rivera Bonnie Spring, Susan Michie, and David A Asch. 2015. Building new computational models to support health behavior change and maintenance: new opportunities in behavioral research. Translational Behavioral Medicine (2015), 1-12.

[81] Donna Spruijt-Metz, Eric Hekler, Niilo Saranummi, Stephen Intille, Ilkka Korhonen, Wendy Nilsen, Daniel E Rivera, Bonnie Spring, Susan Michie, David A Asch, et al. 2015. Building new computational models to support health behavior change and maintenance: new opportunities in behavioral research. Translational behavioral medicine 5, 3 (2015), 335-346.

[82] James Tamerius, Martha I Nelson, Steven Z Zhou, CÃlcile Viboud, Mark A Miller, and Wladimir J Alonso. 2011. Global influenza seasonality: reconciling patterns across temperate and tropical regions. Environmental health perspectives 119, 4 (2011), 439.

[83] Rannie Teodoro and Mor Naaman. 2013. Fitter with Twitter: Understanding Personal Health and Fitness Activity in Social Media.. In ICWSM.

[84] Christopher Torrence and Gilbert P Compo. 1998. A practical guide to wavelet analysis. Bulletin of the American Meteorological society 79, 1 (1998), 61-78.

[85] Michelle Tucker and Marla Reicks. 2002. Exercise as a gateway behavior for healthful eating among older adults: an exploratory study. Fournal of Nutrition Education and Behavior 34 (2002), S14-S19.

[86] Benjamin C Turner, Emma Jenkins, David Kerr, Robert S Sherwin, and David A Cavan. 2001. The effect of evening alcohol consumption on next-morning glucose control in type 1 diabetes. Diabetes Care 24, 11 (2001), 1888-1893.

[87] Venmo. 2017. Venmo API. https://venmo.com/api/v5/public. (2017). Accessed: 2017-07-06.

[88] Akkapon Wongkoblap, Miguel A Vadillo, and Vasa Curcin. 2017. Researching Mental Health Disorders in the Era of Social Media: Systematic Review. Journal of Medical Internet Research 19, 6 (2017), e228.

Received April 2017; revised July 2017; accepted November 2017 